\title{
PERIODIC TWISTS OF GL 3 -AUTOMORPHIC FORMS
}

\author{
EMMANUEL KOWALSKI ${ }^{1}$, YONGXIAO LIN ${ }^{2}$, PHILIPPE MICHEL ${ }^{2}$ and \\ WILL SAWIN ${ }^{3}$ \\ ${ }^{1}$ ETHZ, Rämistrasse 101, 8092 Zürich, Switzerland; \\ email: kowalski@math.ethz.ch \\ ${ }^{2}$ EPFL/MATH/TAN, Station 8, CH-1015 Lausanne, Switzerland; \\ email: yongxiao.lin@epfl.ch,philippe.michel@epfl.ch \\ ${ }^{3}$ Columbia University, USA; \\ email: sawin@math.columbia.edu
}

Received 14 May 2019; accepted 11 January 2020

\begin{abstract}
We prove that sums of length about $q^{3 / 2}$ of Hecke eigenvalues of automorphic forms on $\mathrm{SL}_{3}(\mathbf{Z})$ do not correlate with $q$-periodic functions with bounded Fourier transform. This generalizes the earlier results of Munshi and Holowinsky-Nelson, corresponding to multiplicative Dirichlet characters, and applies, in particular, to trace functions of small conductor modulo primes.
\end{abstract}

2010 Mathematics Subject Classification: 11F55, 11M41, 11L07 (primary); 11T23, 32N10 (secondary)

\section{Introduction}

Let $\varphi$ be a cuspform for $\mathrm{SL}_{3}(\mathbf{Z})$ which is an eigenfunction of all Hecke operators. For any prime number $q$ and any primitive Dirichlet character $\chi$ modulo $q$, we can then define the twisted $L$-function $L(\varphi \otimes \chi, s)$, which is an entire function satisfying a functional equation relating $s$ to $1-s$. In a recent breakthrough, Munshi $[20,21]$ solved the subconvexity problem for these twisted $L$-functions $L(\varphi \otimes \chi, s)$ in the conductor aspect.

THEOREM 1.1 (Munshi). Let $s$ be a complex number such that $\mathfrak{R e} s=1 / 2$. For any prime $q$, any primitive Dirichlet character $\chi$ modulo $q$ and for any $\varepsilon>0$, we have

$$
L(\varphi \otimes \chi, s) \ll q^{3 / 4-1 / 308+\varepsilon},
$$

where the implied constant depends on $\varphi$, $s$ and $\varepsilon$.

(c) The Author(s) 2020. This is an Open Access article, distributed under the terms of the Creative Commons Attribution licence (http://creativecommons.org/licenses/by/4.0/), which permits unrestricted re-use, distribution, and reproduction in any medium, provided the original work is properly cited. 
This result was recently analyzed in depth by Holowinsky and Nelson [12], who discovered a remarkable simplification (and strengthening) of Munshi's ideas. They proved the following.

THEOREM 1.2 (Holowinsky-Nelson). With notation and assumptions as in Theorem 1.1, we have

$$
L(\varphi \otimes \chi, s) \ll q^{3 / 4-1 / 36+\varepsilon},
$$

where the implied constant depends on $\varphi, s$ and $\varepsilon$.

REMARK 1. We mention further variants, simplifications and improvements by Aggarwal et al. [1], Holowinsky et al. [11], Lin [17] and Sun and Zhao [23].

Let $(\lambda(m, n))$ denote the Hecke eigenvalues of $\varphi$. By the approximate functional equation for the twisted $L$-functions, the bound (1.2) is essentially equivalent to the bound

$$
\sum_{n \geqslant 1} \lambda(1, n) \chi(n) V\left(\frac{n}{q^{3 / 2}}\right) \ll q^{3 / 2-\delta},
$$

for $\delta<1 / 36$, where $V$ is any smooth compactly supported function and the implied constant depends on $\varphi, \delta$ and $V$.

From the perspective of such sums, motivated by the previous work of Fouvry et al. [6], which relates to automorphic forms on $\mathrm{GL}_{2}$, it is natural to ask whether this bound (1.3) holds when $\chi$ is replaced by a more general trace function $K$ : $\mathbf{F}_{q} \rightarrow \mathbf{C}$. Our main result shows that this is the case and, in fact, extends the result to a much wider range of $q$-periodic functions by obtaining estimates only in terms of the size of the discrete Fourier transform modulo $q$.

Precisely, for any function $V$ with compact support on $\mathbf{R}$, we set

$$
S_{V}(K, X):=\sum_{n \geqslant 1} \lambda(1, n) K(n) V\left(\frac{n}{X}\right) .
$$

We will assume that $V: \mathbf{R} \rightarrow \mathbf{C}$ satisfies the following conditions for some parameter $Z \geqslant 1$ :

$$
\operatorname{supp}(V) \subset] 1,2\left[\quad \text { and } \quad V^{(i)}(x) \ll Z^{i} \quad \text { for all } i \geqslant 0,\right.
$$

where the implied constant depends only on $i$.

For any integer $q \geqslant 1$ and any $q$-periodic function $K: \mathbf{Z} \rightarrow \mathbf{C}$, we denote by

$$
\widehat{K}(n)=\frac{1}{q^{1 / 2}} \sum_{x \in \mathbf{F}_{q}} K(x) e\left(\frac{n x}{q}\right),
$$


for $n \in \mathbf{Z}$, its (unitarily normalized) discrete Fourier transform modulo $q$. We write $\|\widehat{K}\|_{\infty}$ for the maximum of $|\widehat{K}(n)|$ for $n \in \mathbf{Z}$. We then have the discrete Fourier inversion formula

$$
K(x)=\frac{1}{q^{1 / 2}} \sum_{n \in \mathbf{F}_{q}} \widehat{K}(n) e\left(-\frac{n x}{q}\right)
$$

for $x \in \mathbf{Z}$.

Our main result is a general bound for (1.4) which matches precisely the bound of Holowinsky and Nelson [12] in the case of a multiplicative character.

THEOREM 1.3. Let $\varphi$ be an $\mathrm{SL}_{3}(\mathbf{Z})$-invariant Hecke eigencuspform with Hecke eigenvalues $(\lambda(m, n))$. Let $q$ be a prime number and $K: \mathbf{Z} \rightarrow \mathbf{C}$ be a q-periodic function. Let $V$ be a smooth, compactly supported function satisfying (1.5) for some $Z \geqslant 1$. Assume that

$$
Z^{2 / 3} q^{4 / 3} \leqslant X \leqslant Z^{-2} q^{2}
$$

For any $\varepsilon>0$, we have

$$
S_{V}(K, X) \ll\|\widehat{K}\|_{\infty} Z^{10 / 9} q^{2 / 9+\varepsilon} X^{5 / 6},
$$

where the implied constant depends only on $\varepsilon, \varphi$ and the implicit constants in (1.5).

REMARK 2. (1) Suppose that we vary $q$ and apply this bound with functions $K$ modulo $q$ that have absolutely bounded Fourier transforms. Take $X=q^{3 / 2}$. We then obtain the bound

$$
S_{V}\left(K, q^{3 / 2}\right) \ll Z^{10 / 9} q^{3 / 2-1 / 36+\varepsilon}
$$

for any $\varepsilon>0$.

(2) For the bound (1.7) to be nontrivial (that is, assuming $K$ to be absolutely bounded, better than $X$ ), it is enough that

$$
X \geqslant Z^{20 / 3} q^{4 / 3+\delta}
$$

for some $\delta>0$.

(3) As in the paper [8] of Fouvry, Kowalski, Michel, Raju, Rivat and Soundararajan, where the main estimate is also phrased in Fourier-theoretic terms only, the motivating examples of functions $K$ satisfying uniform 
bounds on their Fourier transforms are the trace functions of suitable $\ell$-adic sheaves modulo $q$. (Although the size of $K$ enters in [8] as well as that of its Fourier transform.) The simplest example is $K(n)=\chi(n)$, which recovers the bound of Munshi (up to the value of the exponent) and HolowinskyNelson, since the values of the Fourier transform are normalized Gauss sums of modulus $\leqslant 1$. We recall some other examples in Section 3.

We can deduce from Theorem 1.3 a weak but nontrivial bound for the first moment of the twisted central $L$-values, with an additional twist by a discrete Mellin transform. We first recall the definition

$$
\mathrm{Kl}_{3}(n)=\frac{1}{q} \sum_{\substack{x, y, z \in \mathbf{F}_{q}^{\times} \\ x y z=n}} e\left(\frac{x+y+z}{q}\right)
$$

for a hyper-Kloosterman sum with two variables modulo a prime $q$.

COROLlaRY 1.4. Let $\varphi$ be an $\mathrm{SL}_{3}(\mathbf{Z})$-invariant cuspidal Hecke eigenform with Hecke eigenvalues $(\lambda(m, n))$. Let $q$ be a prime number and let $\chi \mapsto M(\chi)$ be a function of Dirichlet characters modulo $q$.

Let $K$ and $L$ be the $q$-periodic functions defined by $K(0)=L(0)=0$ and

$$
\begin{aligned}
& K(n)=\frac{q^{1 / 2}}{q-1} \sum_{\chi(\bmod q)} \chi(n) M(\chi) \\
& L(n)=\frac{1}{q^{1 / 2}} \sum_{x \in \mathbf{F}_{q}} K(x) \mathrm{Kl}_{3}(n x)
\end{aligned}
$$

for $n$ coprime to $q$. We then have

$$
\frac{1}{q-1} \sum_{\chi(\bmod q)} M(\chi) L(\varphi \otimes \chi, 1 / 2) \ll\left(\|\widehat{K}\|_{\infty}+\|\widehat{L}\|_{\infty}\right) q^{2 / 9+\varepsilon},
$$

for any $\varepsilon>0$, where the implied constant depends on $\varphi$ and $\varepsilon$.

A further natural application concerns the symmetric square lift of a $\mathrm{GL}_{2}$ cuspform of level 1. Precisely, let $\psi$ be a cuspidal Hecke eigenform for $\mathrm{SL}_{2}(\mathbf{Z})$ with Hecke eigenvalues $(\lambda(n))_{n \geqslant 1}$. We have the following.

COROLlary 1.5. Let $K$ and $V$ be as above and assume that $Z^{2 / 3} q^{4 / 3} \leqslant X \leqslant$ $Z^{-2} q^{2}$. Then, for any $\varepsilon>0$, we have

$$
\sum_{n \geqslant 1} \lambda\left(n^{2}\right) K(n) V\left(\frac{n}{X}\right) \ll\|\widehat{K}\|_{\infty} Z^{10 / 9} q^{2 / 9+\varepsilon} X^{5 / 6}+\|K\|_{\infty} Z^{1 / 3} q^{2 / 3} X^{1 / 2+\varepsilon},
$$


where the implied constant depends only on $\varepsilon, \psi$ and the implicit constants in (1.5).

REMARK 3. As pointed out to us by Blomer, when $K=\chi$ is a Dirichlet character, a stronger bound should be available: for $\chi$ quadratic, one has (see [2]) the stronger subconvex bound for the central value

$$
L\left(\operatorname{sym}_{2}(\psi) \otimes \chi, s\right) \ll_{s} q^{3 / 4-1 / 8+o(1)}, \quad \mathfrak{R e} s=1 / 2 .
$$

This would amount to a bound of the shape

$$
\sum_{n \geqslant 1} \lambda\left(n^{2}\right) \chi(n) V\left(\frac{n}{q^{3 / 2}}\right) \ll_{Z} q^{3 / 2-1 / 8+\varepsilon} .
$$

The bound (1.8) actually extends to any character $\chi(\bmod q)$ by the same method, using the Petrow-Young variant of the Conrey-Iwaniec method [3, 22]. However, since this approach uses positivity of central values, it is not entirely clear yet whether this could be extended to general trace functions.

From this corollary, one can easily derive an estimate for twists of the arithmetic function $\lambda(n)^{2}=|\lambda(n)|^{2}$, which is related to $\lambda\left(n^{2}\right)$ by the convolution identity

$$
\lambda(n)^{2}=\sum_{a b=n} \lambda\left(a^{2}\right)
$$

However, in terms of $L$-functions, a straightforward estimate concerns sums of length close to $q^{2}$ and not $q^{3 / 2}$ anymore (it amounts, when $K=\chi$, to a subconvexity estimate for $L\left(f \otimes f \otimes \chi, \frac{1}{2}\right)$, which results directly from the factorization of this $L$-function of degree 4 ).

One can however recover a bound for sums of length about $q^{3 / 2}$ with more work, and here we require that $K$ be a trace function (more precisely, a nonexceptional trace function, in the sense of [4, page 1686]).

COROLlary 1.6. Let $V$ be as above. Let $K$ be the trace function of an $\ell$-adic sheaf $\mathcal{F}$ modulo $q$ which is a geometrically irreducible middle-extension sheaf, pure of weight 0 , on the affine line over $\mathbf{F}_{q}$. Assume that the sheaf $\mathcal{F}$ is not geometrically isomorphic to the tensor product $\mathcal{L}_{\psi} \otimes \mathcal{L}_{\chi}$ of an Artin-Schreier sheaf and a Kummer sheaf.

If $Z^{-4 / 3} q^{4 / 3+8 \gamma / 3} \leqslant X \leqslant Z^{-2} q^{2}$, then we have

$$
\sum_{n \geqslant 1} \lambda(n)^{2} K(n) V\left(\frac{n}{X}\right) \ll X^{2 / 3+\varepsilon} q^{1 / 3}+Z^{5 / 6} X^{7 / 8+\varepsilon} q^{1 / 6}+X^{1+\varepsilon} q^{-\gamma}
$$


for any $\varepsilon>0$, where the implied constant depends only on $\psi, \varepsilon$ and the conductor $\mathbf{c}(\mathcal{F})$ of $\mathcal{F}$.

REMARK 4. (1) Suppose that $Z$ is fixed. The estimate is then nontrivial as long as $X \gg q^{4 / 3+\delta}$; for $X=q^{3 / 2}$, it saves a factor $q^{1 / 48}$ over the trivial bound.

(2) The assumption that $\mathcal{F}$ is not exceptional means intuitively that $K$ is not proportional to the product of an additive and a multiplicative character modulo $q$. We then have, in particular,

$$
\|K\|_{\infty}+\|\widehat{K}\|_{\infty} \ll 1,
$$

where the implied constant depends only on the conductor of $\mathcal{F}$.

REMARK 5. (1) The reader may wonder why this paper is much shorter than [6] and (with the exception of Corollary 1.6) requires much less input from algebraic geometry in the case of trace functions. One reason is that we are considering (essentially) sums of length $q^{3 / 2}$, whereas the coefficient functions $K$ are $q$-periodic. This means that periodicity properties of the summand $K(n)$ have a nontrivial effect, whereas they do not for the sums of length about $q$ which are considered in [6] in the context of $\mathrm{GL}_{2}$.

Moreover, observe that an analogue of Theorem 1.3, with an estimate that depends (in terms of $K$ ) only on the size of the Fourier transform $\widehat{K}$, is false in the setting of [6], that is, for sums

$$
\sum_{n \geqslant 1} \lambda(n) K(n) V\left(\frac{n}{X}\right)
$$

with $X$ of size about $q$, where $\lambda(n)$ are the Hecke eigenvalues of a cuspform $\psi$ for $\mathrm{SL}_{2}(\mathbf{Z})$ (as in Corollary 1.5). Indeed, if we take $X=q$ and define $K$ to be the $q$-periodic function that coincides with the (real-valued) function $n \mapsto \lambda(n)$ for $1 \leqslant n \leqslant q$, then $K$ has discrete Fourier transform of size $\ll \log q$ by the well-known Wilton estimate (see, for example, [13, Theorem 5.3], when $\psi$ is holomorphic), and, yet,

$$
\sum_{n \leqslant q} K(n) \lambda(n)=\sum_{n \leqslant q}|\lambda(n)|^{2} \asymp q
$$

by the Rankin-Selberg method.

On the other hand, the same bound of Wilton combined with discrete Fourier inversion implies quickly that if $K$ is any $q$-periodic function, then

$$
\sum_{n \leqslant q^{3 / 2}} \lambda(n) K(n) \ll q^{1+1 / 4+\varepsilon}\|\widehat{K}\|_{\infty}
$$


for any $\varepsilon>0$. However, the natural length for applications is $q$ in the $\mathrm{GL}_{2}$ case.

(2) The most obvious function $K$ for which Theorem 1.3 gives trivial results is an additive character $K(n)=e(a n / q)$ for some integer $a \in \mathbf{Z}$ since the Fourier transform takes one value of size $q^{1 / 2}$. However, a useful estimate also exists in this case: Miller [18] has proved that

$$
\sum_{n \geqslant 1} \lambda(1, n) e(\alpha n) V\left(\frac{n}{X}\right) \ll_{\varphi, Z} X^{3 / 4+\varepsilon}
$$

for $X \geqslant 2$, any $\alpha \in \mathbf{R}$ and any $\varepsilon>0$, where the implied constant is independent of $\alpha$. This is the generalization to $\mathrm{GL}_{3}$ of the bound of Wilton mentioned in the first remark.

(3) Using either the functional equation for the $L$-functions $L(\varphi \otimes \chi, s)$ or the Voronoi summation formula, one can show that the estimate of Miller implies a bound of the shape

$$
S_{V}\left(\mathrm{Kl}_{2}(a \cdot ; q), X\right) \ll_{\varphi, Z}(q X)^{\varepsilon} X^{1 / 4} q^{3 / 4}
$$

for any $\varepsilon>0$, where

$$
\mathrm{Kl}_{2}(n ; q)=\frac{1}{q^{1 / 2}} \sum_{x \in \mathbf{F}_{q}^{\times}} e_{q}(\bar{x}+n x)
$$

is a normalized Kloosterman sum. This bound is nontrivial as long as $X \geqslant q$. Since $\mathrm{Kl}_{2}$ is a trace function that is bounded by 2 and has Fourier transform bounded by 1 , this gives (in a special case) a stronger bound than what follows from Theorem 1.3.

(4) Remark (2) suggests a direct approach by the discrete Fourier inversion formula, which gives

$$
\sum_{n \leqslant X} \lambda(1, n) K(n)=\frac{1}{\sqrt{q}} \sum_{0 \leqslant h<q} \widehat{K}(h) \sum_{n \leqslant X} \lambda(1, n) e\left(\frac{n h}{q}\right) .
$$

A nontrivial bound for $X \approx q^{3 / 2}$ in terms of $\|\widehat{K}\|_{\infty}$ would then follow from a bound

$$
\sum_{n \leqslant X} \lambda(1, n) e\left(\frac{n h}{q}\right) \ll X^{\alpha}
$$

for additive twists of the Fourier coefficients where $\alpha<2 / 3$. 
Unsurprisingly, in the case of $\mathrm{GL}_{2}$, although we have the best possible estimate of Wilton (with the analogue of $\alpha$ being 1/2), the resulting estimate for a sum of length $q$ is trivial.

The plan of the paper is as follows: we will explain the idea and sketch the key steps of the proof in Section 2. Section 3 recalls the most important examples of trace functions, for which $K$ has small Fourier transform and hence for which Theorem 1.3 is nontrivial. Section 4 presents a key Fouriertheoretic estimate and some reminders concerning automorphic forms and the Voronoi summation formula for $\mathrm{GL}_{3}$. Then the last sections complete the proof of Theorem 1.3 following the outline presented previously and explain how to deduce Corollaries 1.4, 1.5 and 1.6 (the last of which requires further ingredients).

Notation. For any $z \in \mathbf{C}$, we define $e(z)=\exp (2 \pi i z)$. If $q \geqslant 1$, then we denote by $e_{q}(x)$ the additive character modulo $q$ defined by $e_{q}(x)=e(x / q)$ for $x \in \mathbf{Z}$. We often identify a $q$-periodic function defined on $\mathbf{Z}$ with a function on $\mathbf{Z} / q \mathbf{Z}$.

For any finite abelian group $A$, we use the notation $\widehat{f}$ for the unitary Fourier transform defined on the character group $\widehat{A}$ of $A$ by

$$
\widehat{f}(\psi)=\frac{1}{\sqrt{|A|}} \sum_{x \in A} f(x) \psi(x) .
$$

We have then the Plancherel formula $\|f\|_{2}=\|\widehat{f}\|_{2}$, where

$$
\|f\|_{2}=\sum_{x \in A}|f(x)|^{2}, \quad\|\widehat{f}\|_{2}=\sum_{\psi \in \widehat{A}}|\widehat{f}(\psi)|^{2} .
$$

For any integrable function on $\mathbf{R}$, we denote its Fourier transform by

$$
\widehat{V}(y)=\int_{\mathbf{R}} V(x) e(-x y) d x .
$$

We recall the Poisson summation formula when performed with a $q$-periodic function $K$ in addition to a smooth function $V$ with fast decay at infinity: for any $X \geqslant 1$, we have

$$
\sum_{n \in \mathbf{Z}} K(n) V\left(\frac{n}{X}\right)=\frac{X}{q^{1 / 2}} \sum_{h \in \mathbf{Z}} \widehat{K}(h) \widehat{V}\left(\frac{h X}{q}\right) .
$$

This follows directly from the usual Poisson formula and the definition of $\widehat{K}$ after splitting the sum into congruence classes modulo $q$. 


\section{Principle of the proof}

We use a direct generalization of the method of Holowinsky and Nelson [12] that led to Theorem 1.2. Although it was motivated by Munshi's approach, based on the use of the Petersson formula as a tool to express the delta symbol, there is no remaining trace of this point of view; however, we refer to [12, Appendix B] for a detailed and insightful description of the origin of this streamlined method, starting from Munshi's.

2.1. Amplification. The first step is to realize the $q$-periodic function $K$ within a one-parameter family of $q$-periodic functions. Precisely, let $\widehat{K}$ be the Fourier transform of $K$ (see (1.6)) and define

$$
\widehat{K}(z, h):= \begin{cases}\widehat{K}(z) e_{q}(-h \bar{z}) & q \nmid z \\ \widehat{K}(0) & q \mid z\end{cases}
$$

for $(z, h) \in \mathbf{Z}^{2}$. Then put

$$
K(n, h)=\frac{1}{q^{1 / 2}} \sum_{z \in \mathbf{F}_{q}^{\times}} \widehat{K}(z, h) e_{q}(-n z)
$$

for $(n, h) \in \mathbf{Z}^{2}$. By the discrete Fourier inversion formula, we have

$$
K(n, 0)=K(n)-\frac{\widehat{K}(0)}{q^{1 / 2}} .
$$

More generally, for any probability measure $\varpi$ on $\mathbf{F}_{q}^{\times}$, the average

$$
K_{\varpi}(n, h)=\sum_{l \in \mathbf{F}_{q}^{\times}} \varpi(l) K(n, \bar{l} h)
$$

satisfies $K_{\varpi}(n, 0)=K(n)-\frac{\widehat{K}(0)}{q^{1 / 2}}$. It follows that, for any parameter $H \geqslant 1$, we can express the sum $S_{V}(K, X)$ as the difference of double sums

$$
S_{V}(K, X)=\sum_{l \in \mathcal{L}} \varpi(l) \sum_{|h| \leqslant H} S_{V}(K(\cdot, h \bar{l}), X)-\sum_{l \in \mathcal{L}} \varpi(l) \sum_{0<|h| \leqslant H} S_{V}(K(\cdot, h \bar{l}), X),
$$

up to an error $\ll X / q^{1 / 2}$. We write this difference as

$$
S_{V}(K, X)=\mathcal{F}-\mathcal{O},
$$

say. One then needs to select a suitable probability measure $\varpi$, and then the two terms are handled by different methods. It should be emphasized that no main 
term arises (which would have to be canceled in the difference between the two terms).

REMARK 6. The argument is reminiscent of the amplification method, the function $K(n)=K(n, 0)$ being 'amplified' (up to a small error) within the family $(K(n, h))_{|h| \leqslant H}$.

2.2. Bounding $\mathcal{F}$. As in [12], we consider a probability measure $\varpi$ corresponding to a product structure: we average over pairs $(p, l)$ of primes such that $p \sim P$ and $l \sim L$ and take $\varpi(x)$ proportional to the number of representations $x=\bar{p} l(\bmod q)$, where $p \sim P$ and $l \sim L$ are primes (their sizes being parameters $1 \leqslant P, L<q / 2$ to be chosen later).

The treatment of $\mathcal{F}$ is essentially the same as in $[12,17]$. By applying the Poisson summation formula to the $h$-variable, with dual variable $r$, we see that the function

$$
(n, r, p, l) \mapsto \widehat{K}(-p \overline{l r}) \lambda(1, n) e_{q}(n p \overline{l r})
$$

appear. We then appeal to the classical 'reciprocity law' for additive exponentials, namely

$$
e_{q}(n p \overline{l r}) \approx e_{r l}(-n p \bar{q}),
$$

trading the modulus $q$ for the modulus $r l$, which will be significantly smaller than $q$. We then apply the Voronoi summation formula for the cuspform $\varphi$ on the $n$ variable (this is the only real automorphic input), which transforms the additive phase $e_{r l}(-n p \bar{q})$ into Kloosterman sums of modulus $r l$. We then obtain further cancellation by smoothing out the resulting variable (dual to $n$ ) by using CauchySchwarz and detecting cancellations on averages of products of Kloosterman sums, where the product structure of the averaging set is essential.

In this part of the argument, the coefficient function $K$ plays a very little role, and we could just more or less quote the corresponding statements in $[12,17]$, if the parameter $Z$ was fixed. Since we wish to keep track of its behavior (for the purpose of flexibility for potential applications), we have to go through the computations anew. This is done in detail in Section 6.

2.3. Bounding $\mathcal{O}$. In the sum $\mathcal{O}$, with the averaging performed in the same way as for $\mathcal{F}$, the key point is that the $n$-variable in the sum $S_{V}(K(\cdot, h \bar{l}, X))$ is very long compared to $q$. We apply Cauchy's inequality to smooth it, keeping the other variables $h, p, l$ inside, thus eliminating the automorphic coefficients $\lambda(1, n)$ (for which we only require average bounds, which we borrow from the Rankin-Selberg theory, our second important automorphic input). This leads 
quickly to the problem of estimating the sum

$$
\sum_{p_{1}, h_{1}, l_{1}, p_{2}, h_{2}, l_{2}} \sum_{n \sim X} K\left(n, h_{1} p_{1} \bar{l}_{1}\right) \overline{K\left(n, h_{2} p_{2} \bar{l}_{2}\right)} .
$$

We apply the Poisson formula in the $n$-variable; since $X$ is typically much larger than $q$, only the zero frequency in the dual sum contributes. This yields a key sum of the shape

$$
\begin{aligned}
& \sum_{p_{1}, h_{1}, l_{1}, p_{2}, h_{2}, l_{2}} \sum_{u \in \mathbf{F}_{q}^{\times}}|\widehat{K}(u)|^{2} e_{q}\left(\left(h_{1} p_{1} \bar{l}_{1}-h_{2} p_{2} \bar{l}_{2}\right) u^{-1}\right) \\
& =\sum_{p_{1}, h_{1}, l_{1}, p_{2}, h_{2}, l_{2}} K_{2}\left(h_{1} p_{1} \bar{l}_{1}-h_{2} p_{2} \bar{l}_{2}\right),
\end{aligned}
$$

say.

When $K$ is a multiplicative character, as in the work of Holowinsky-Nelson, the proof is essentially finished then since $\widehat{K}(u)$ is a normalized Gauss sum, with a constant modulus; hence, $K_{2}$ is simply a Ramanujan sum, which we can evaluate explicitly.

In general, we obtain cancellation using a very general Fourier-theoretic bound for general bilinear forms

$$
\sum_{m \in \mathbf{F}_{q}} \sum_{n \in \mathbf{F}_{q}} \alpha_{m} \beta_{n} K_{2}(m-n)
$$

which involves only $L^{2}$-norm bounds for the coefficients and $L^{\infty}$-norm bounds for the Fourier transform of $K_{2}$ (see Proposition 4.1). The latter, it turns out, is essentially $|\widehat{K}|^{2}$, and we can obtain a good estimate purely in terms of $\|\widehat{K}\|_{\infty}$. This part of the argument is performed in Section 7.

\section{Examples of trace functions}

Theorem 1.3 certainly applies to 'random' $q$-periodic functions $K: \mathbf{Z} \rightarrow \mathbf{C}$, for all reasonable meanings of the word 'random'; but the basic motivating examples in number theory are often provided by trace functions. Since there are by now a number of surveys and discussions of important examples (see, for example, [6, Section 10], [8, Section 2.2] or [5]), we only recall some of them for concreteness.

- If $r \geqslant 1$ is a fixed integer and $\chi_{1}, \ldots, \chi_{r}$ are distinct nontrivial Dirichlet characters modulo $q$, of order $d_{i} \geqslant 2$, and if $f_{1}, \ldots, f_{r}, g$ are polynomials 
in $\mathbf{Z}[X]$ such that either $\operatorname{deg}(g(\bmod q)) \geqslant 2$ or one of the $f_{i}(\bmod q)$ is not proportional to a $d_{i}$ th power in $\overline{\mathbf{F}}_{q}[X]$, then

$$
K(n)=\chi_{1}\left(f_{1}(n)\right) \cdots \chi_{r}\left(f_{r}(n)\right) e\left(\frac{g(n)}{q}\right)
$$

has Fourier transform of size bounded only in terms of $r$ and the degrees of the polynomials $f_{i}$ and $g$. (This is a consequence of the Weil bounds for exponential sums in one variable.)

Moreover (as is relevant only for Corollary 1.6 in this paper), $K$ is a trace function, and it is nonexceptional, unless $g$ is of degree $1, r=1$ and $f_{1}$ is of degree $\leqslant 1$.

- Let $r \geqslant 2$. Define $\mathrm{Kl}_{r}(0)=0$ and

$$
\mathrm{Kl}_{r}(n)=\frac{1}{q^{(r-1)-2}} \sum_{\substack{x_{1}, \ldots, x_{r} \in \mathbf{F}_{q} \\ x_{1} \cdots, x_{r}=n}} e\left(\frac{x_{1}+\cdots+x_{r}}{q}\right)
$$

for $n \in \mathbf{F}_{q}^{\times}$(these are hyper-Kloosterman sums). Then $\left\|\widehat{\mathrm{K}} \mathrm{l}_{r}\right\|_{\infty} \leqslant c_{r}$, where $c_{r}$ depends only on $r$ (this depends on Deligne's general proof of the Riemann Hypothesis over finite fields and on the construction and basic properties of Kloosterman sheaves).

For all $r \geqslant 2$, the function $\mathrm{Kl}_{r}$ is a trace function of a nonexceptional sheaf.

We also mention one important principle: if $K$ is the trace function of a Fourier sheaf $\mathcal{F}$ (in the sense of [14]), then $\widehat{K}$ is also such a function for a sheaf $\operatorname{FT}(\mathcal{F})$; moreover, if $\mathcal{F}$ has conductor $c$ (in the sense of [6]), then $\mathrm{FT}(\mathcal{F})$ has conductor $\leqslant 10 c^{2}$, and, in particular, $\|\widehat{K}\|_{\infty} \leqslant 10 c^{2}$.

Finally, one example that is not usually discussed explicitly (formally, because it arises from a skyscraper sheaf) is when $K(n)=q^{1 / 2} \delta_{n=a(\bmod q)}$ is the $L^{2}$ normalized delta function at a point $a \in \mathbf{Z}$. In this case, the Fourier transform is an additive character, and hence is bounded by one; dividing by $q^{1 / 2}$, we obtain the bound

$$
\sum_{\substack{n \geqslant 1 \\ n \equiv a(\bmod q)}} \lambda(1, n) V\left(\frac{n}{X}\right) \ll Z^{10 / 9} q^{-5 / 18+\varepsilon} X^{5 / 6},
$$

under the assumptions of Theorem 1.3; in particular, if $X=q^{3 / 2}$ and $V$ satisfies (1.5) for $Z=1$, we get

$$
\sum_{\substack{n \geqslant 1 \\ n \equiv a(\bmod q)}} \lambda(1, n) V\left(\frac{n}{q^{3 / 2}}\right) \ll q^{35 / 36+\varepsilon}
$$


for any $\varepsilon>0$. Note that, under the generalized Ramanujan-Petersson conjecture $\lambda(1, n) \ll n^{\varepsilon}$, we would obtain the stronger bound $q^{1 / 2+\varepsilon}$ (and knowing the approximation $\lambda(1, n) \ll n^{\theta}$ for some $\theta<1 / 3$ would be enough to get a nontrivial bound). We discuss this case in further detail in Remark 10, in the context of Corollary 1.4.

\section{Preliminaries}

4.1. A Fourier-theoretic estimate. A key estimate in Section 7 will arise from the following general bound (special cases of which have appeared before, for example, in the case of multiplicative characters for problems concerning sums over sumsets).

Proposition 4.1. Let A be a finite abelian group, with group operation denoted additively. Let $\alpha, \beta$ and $K$ be functions from $A$ to $\mathbf{C}$. We have

$$
\left|\sum_{m, n \in A} \alpha(m) \beta(n) K(m-n)\right| \leqslant|A|^{1 / 2}\|\widehat{K}\|_{\infty}\|\alpha\|_{2}\|\beta\|_{2} .
$$

Proof. Using orthogonality of characters, we write

$$
\sum_{m, n \in A} \alpha(m) \beta(n) K(m-n)=\sum_{m, n, h \in A} \alpha(m) \beta(n) K(h) \frac{1}{|A|} \sum_{\psi \in \widehat{A}} \psi(h-(m-n)) .
$$

Moving outside the sum over $\psi$, this is equal to

$$
|A|^{1 / 2} \sum_{\psi \in \widehat{A}} \widehat{\alpha}\left(\psi^{-1}\right) \widehat{\beta}(\psi) \widehat{K}(\psi),
$$

whose absolute value is

$$
\leqslant|A|^{1 / 2}\|\widehat{K}\|_{\infty} \sum_{\psi \in \widehat{A}}\left|\widehat{\alpha}\left(\psi^{-1}\right) \widehat{\beta}(\psi)\right| \leqslant|A|^{1 / 2}\|\widehat{K}\|_{\infty}\|\alpha\|_{2}\|\beta\|_{2},
$$

by the Cauchy-Schwarz inequality and the discrete Plancherel formula.

4.2. Background on $\mathbf{G L}_{3}$-cuspforms. We refer to [10, Ch. 6] for notations. Let $\varphi$ be a cuspform on $\mathrm{GL}_{3}$ with level 1 and with Langlands parameters $\mu=\left(\mu_{1}\right.$, $\left.\mu_{2}, \mu_{3}\right) \in \mathbf{C}^{3}$. We denote by $(\lambda(m, n))_{m, n \neq 0}$ its Fourier-Whittaker coefficients and assume that $\varphi$ is an eigenform of the Hecke operators $T_{n}$ and $T_{n}^{*}$, normalized so that $\lambda(1,1)=1$. The eigenvalue of $T_{n}$ is then $\lambda(1, n)$ for $n \geqslant 1$. 
Let $\theta_{3}=5 / 14$. The archimedean parameters and the Hecke eigenvalues are bounded individually by

$$
\left|\mathfrak{R e}\left(\mu_{i}\right)\right| \leqslant \theta_{3}, \quad|\lambda(1, p)| \leqslant 3 p^{\theta_{3}}
$$

for any $i$ and any prime number $p$ (see [15]).

Average estimates follow from the Rankin-Selberg method. We have

$$
\sum_{1 \leqslant n \leqslant X}|\lambda(1, n)|^{2} \ll X^{1+\varepsilon}
$$

and

$$
\sum_{1 \leqslant m^{2} n \leqslant X} m|\lambda(m, n)|^{2} \ll X^{1+\varepsilon},
$$

for $X \geqslant 2$ and any $\varepsilon>0$, where the implied constant depends only on $\varphi$ and $\varepsilon$. (See [19] and [21, Lemma 2].)

The key analytic feature of $\mathrm{GL}_{3}$-cuspforms that we use (as in previous works) is the Voronoi summation formula for $\varphi$ (originally due to Miller-Schmid, and Goldfeld-Li independently). Since our use of the 'archimedean' part of the formula is quite mild, we use the same compact formulation as in [12, Section 2.3], where references are given.

Let $q \geqslant 1$ be an integer (not necessarily prime). For $n \in \mathbf{Z}$, we denote

$$
\mathrm{Kl}_{2}(n ; q)=\frac{1}{\sqrt{q}} \sum_{x \in(\mathbf{Z} / q \mathbf{Z})^{\times}} e\left(\frac{n x+\bar{x}}{q}\right),
$$

where $\bar{x}$ is the inverse of $x$ modulo $q$.

LEMMA 4.2 (Voronoi summation formula). For $\sigma \in\{-1,1\}$, there exist functions $\mathcal{G}^{\sigma}$, meromorphic on $\mathbf{C}$, holomorphic for $\mathfrak{R e}(s)>\theta_{3}$, with polynomial growth in vertical strips $\mathfrak{R e}(s) \geqslant \alpha$ for any $\alpha>\theta_{3}$ such that the following properties hold.

Let $a$ and $q \geqslant 1$ be coprime integers, let $X>0$ and let $V$ be a smooth function on $] 0,+\infty[$ with compact support. We have

$$
\begin{aligned}
& \sum_{n \geqslant 1} \lambda(1, n) e_{q}(\text { an }) V\left(\frac{n}{X}\right) \\
& \quad=q^{3 / 2} \sum_{\sigma \in\{-1,1\}} \sum_{n \geqslant 1} \sum_{m \mid q} \frac{\lambda(n, m)}{n m^{3 / 2}} \mathrm{Kl}_{2}\left(\sigma n \bar{a} ; \frac{q}{m}\right) \mathcal{V}_{\sigma}\left(\frac{m^{2} n}{q^{3} / X}\right),
\end{aligned}
$$

where

$$
\nu_{\sigma}(x)=\frac{1}{2 \pi i} \int_{(1)} x^{-s} \mathcal{G}^{\sigma}(s+1)\left(\int_{0}^{+\infty} V(y) y^{-s} \frac{d y}{y}\right) d s .
$$


Note that the functions $\mathcal{G}^{\sigma}$ depend (only) on the archimedean parameters of $\varphi$. We record some properties of the functions $\mathcal{V}_{\sigma}(x)$; for $Z$ fixed, they are already explained in [12, Section 2.3].

LeMmA 4.3. Let $\sigma \in\{-1,1\}$. For any $j \geqslant 0$, any $A \geqslant 1$ and any $\varepsilon>0$, we have

$$
x^{j} \mathcal{V}_{\sigma}^{(j)}(x) \ll \min \left(Z^{j+1} x^{1-\theta_{3}-\varepsilon}, Z^{j+5 / 2+\varepsilon}\left(\frac{Z^{3}}{x}\right)^{A}\right)
$$

for $x>0$, where the implied constant depends on $(j, A, \varepsilon)$. Moreover, for $x \geqslant 1$, we have

$$
x^{j} \mathcal{V}_{\sigma}^{(j)}(x) \ll x^{2 / 3} \min \left(Z^{j}, x^{j / 3}\right)
$$

where the implied constant depends on $j$.

Proof. The first inequality in the first bound follows by shifting the contour in $\mathcal{V}_{ \pm}(x)$ to $\mathfrak{R e} s=\theta_{3}-1+\varepsilon$, while the second one follows by shifting contour to the far right. The second bound follows from [2, Lemma 6].

In particular, we see from the lemma that the functions $\mathcal{V}_{\sigma}(x)$ decay very rapidly as soon as $x \geqslant X^{\delta} Z^{3}$ for some $\delta>0$.

REMARK 7. The bound $x^{j} \mathcal{V}_{\sigma}^{(j)}(x) \ll Z^{j+1} x^{1-\theta_{3}-\varepsilon}$ can be replaced by $x^{j} \mathcal{V}_{\sigma}^{(j)}(x)$ $\ll Z^{j} x^{1-\varepsilon}$ under the Ramanujan-Selberg conjecture, that is, if $\mathfrak{R e}\left(\mu_{i}\right)=0$ for all $i$.

REMARK 8 . Let $N \geqslant 1$, and define a congruence subgroup $\Gamma_{N} \subset \mathrm{SL}_{3}(\mathbf{Z})$ by

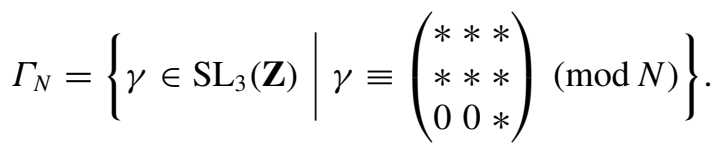

Zhou [25] has established an explicit Voronoi summation formula for $\mathrm{GL}_{3}$ cuspforms that are invariant under $\Gamma_{N}$, for additive twists by $e_{q}(a n)$ when either $(q, N)=1$ or $N \mid q$. It should then be possible to use this formula to generalize Theorem 1.3 to such cuspforms by slight adaptations of the argument below.

\section{Amplification of the trace function}

We now begin the proof of Theorem 1.3. Let $q$ be a prime number and $K$ a $q$-periodic function on $\mathbf{Z}$. Let $\widehat{K}$ be its discrete Fourier transform (1.6), which is also a $q$-periodic function on $\mathbf{Z}$. 
Let $P, L \geqslant 1$ be two parameters to be chosen later, with $2 P<q$ and $2 L<q$. We define auxiliary sets

$$
\begin{aligned}
& \mathrm{P}:=\{p \in[P, 2 P[\mid p \equiv 1(\bmod 4), \text { prime }\} \\
& \mathrm{L}:=\{l \in[L, 2 L[\mid l \equiv 3(\bmod 4), \text { prime }\} .
\end{aligned}
$$

Note that these sets are disjoint. We denote

$$
H=\frac{q^{2} L}{X P} .
$$

In the sequel, we assume that $H \geqslant 1$, that is,

$$
X P \leqslant q^{2} L \text {. }
$$

Let $W$ be a smooth function on $\mathbf{R}$ that satisfies (1.5) with $Z=1$ and, furthermore, $\widehat{W}(0)=1$.

We now use the notations $K(n, h)$ and $\widehat{K}(z, h)$ and the basic amplification idea discussed in Section 2.1 (see (2.1) and (2.2)). We define

$$
\begin{aligned}
\mathcal{F} & =\frac{1}{|\mathrm{P}||\mathrm{L}|} \sum_{p \in \mathrm{P}} \sum_{l \in \mathrm{L}} \sum_{h \in \mathbf{Z}} S_{V}(K(\cdot, h p \bar{l}), X) \widehat{W}\left(\frac{h}{H}\right) \\
& =\frac{1}{|\mathrm{P}||\mathrm{L}|} \sum_{p \in \mathrm{P}} \sum_{l \in \mathrm{L}} \sum_{h \in \mathbf{Z}} \widehat{W}\left(\frac{h}{H}\right) \sum_{n \geqslant 1} \lambda(1, n) K(n, h p \bar{l}) V\left(\frac{n}{X}\right) .
\end{aligned}
$$

Separating the contribution of $h=0$ and applying (2.3), we can write

$$
\mathcal{F}=S_{V}(K, X)+\mathcal{O}+O\left(\frac{q^{\varepsilon}\|\widehat{K}\|_{\infty} X}{q^{1 / 2}}\right),
$$

for any $\varepsilon>0$, where

$$
\mathcal{O}=\frac{1}{|\mathrm{P}||\mathrm{L}|} \sum_{p \in \mathrm{P}} \sum_{l \in \mathrm{L}} \sum_{h \neq 0} \widehat{W}\left(\frac{h}{H}\right) \sum_{n \geqslant 1} \lambda(1, n) K(n, h p \bar{l}) V\left(\frac{n}{X}\right) .
$$

Indeed, the contribution of $h=0$ is

$$
\begin{aligned}
& \frac{1}{|\mathrm{P}||\mathrm{L}|} \sum_{p \in \mathrm{P}} \sum_{l \in \mathrm{L}} S_{V}(K(\cdot, 0), X) \widehat{W}(0) \\
& =S_{V}(K, X)-\frac{\widehat{K}(0)}{|\mathrm{P}||\mathrm{L}| q^{1 / 2}} \sum_{p \in \mathrm{P}} \sum_{l \in \mathrm{L}} \sum_{n \geqslant 1} \lambda(1, n) V\left(\frac{n}{X}\right) \\
& =S_{V}(K, X)+O\left(\frac{\|\widehat{K}\|_{\infty} X^{1+\varepsilon}}{q^{1 / 2}}\right),
\end{aligned}
$$

for any $\varepsilon>0$, by (4.1). 


\section{Evaluation of $\mathcal{F}$}

The evaluation of $\mathcal{F}$ is close to the arguments of [12] and [17, Section 6]. In fact, we could extract the desired bounds from these sources (especially [17]) in the important special case when the parameter $Z$ is fixed as $q$ varies. The reader who is familiar with one of these references may therefore wish to skip the proof of the next proposition in a first reading.

Proposition 6.1. Let $\eta>0$. Assume that

$$
X / Z \geqslant q^{1+\eta}
$$

and

$$
L \leqslant P^{4}
$$

Then for any $\varepsilon>0$, we have

$$
\mathcal{F} \ll q^{\varepsilon}\|\widehat{K}\|_{\infty}\left(\frac{Z^{2} X^{3 / 2} P}{q L^{1 / 2}}+Z^{3 / 2} X^{3 / 4}(q P L)^{1 / 4}\right),
$$

where the implied constant depends on $\varphi, \varepsilon$ and $\eta$.

The remainder of this section is dedicated to the proof of this proposition. We fix $\eta$ satisfying (6.1).

We apply the Poisson summation formula to the sum over $h$ in $\mathcal{F}$, for each $(p, l)$. We obtain

$$
\sum_{h \in \mathbf{Z}} K(n, h p \bar{l}) \widehat{W}\left(\frac{h}{H}\right)=\frac{H}{q^{1 / 2}} \sum_{(r, q)=1} \widehat{K}(-p \bar{l} \bar{r}) e_{q}(n p \bar{l} \bar{r}) W\left(\frac{r}{R}\right),
$$

where

$$
R=q / H=\frac{X P}{q L}
$$

Hence, it follows that

$$
\mathcal{F}=\frac{q^{3 / 2} L}{X P|\mathrm{P}||\mathrm{L}|} \sum_{p \in \mathrm{P}} \sum_{l \in \mathrm{L}} \sum_{(r, q)=1} \widehat{K}(-p \bar{l} \bar{r}) \sum_{n \geqslant 1} e_{q}(n p \bar{l} \bar{r}) \lambda(1, n) V\left(\frac{n}{X}\right) W\left(\frac{r}{R}\right) .
$$

Since $l \leqslant 2 L<q$, we have $(q, r l)=1$ in the sums. By reciprocity, we have

$$
e_{q}(n p \bar{l} \bar{r})=e_{r l}(-n p \bar{q}) e_{q r l}(n p)
$$

for $n \geqslant 1$. 
REMARK 9. Note that for $n \asymp X$, we have

$$
\frac{n p}{q r l} \asymp \frac{X P}{q L X P /(q L)} \asymp 1
$$

so that the additive character $e_{q r l}(n p)$ does not oscillate.

We define

$$
V_{1}(x)=e\left(\frac{x X p}{q r l}\right) V(x)
$$

We can then rephrase the above as

$$
\sum_{n \geqslant 1} \lambda(1, n) e_{r l}(-n p \bar{q}) e_{q r l}(n p) V\left(\frac{n}{X}\right)=\sum_{n \geqslant 1} \lambda(1, n) e_{r l}(-n p \bar{q}) V_{1}\left(\frac{n}{X}\right),
$$

and

$$
\mathcal{F}=\frac{q^{3 / 2} L}{X P|\mathrm{P}||\mathrm{L}|} \sum_{p, l} \sum_{r \geqslant 1} \widehat{K}(-p \bar{l} \bar{r}) W\left(\frac{r}{R}\right) \sum_{n \geqslant 1} \lambda(1, n) e_{r l}(-n p \bar{q}) V_{1}\left(\frac{n}{X}\right) .
$$

Let $\mathcal{F}^{\prime}$ be the contribution to the last expression of those $(p, r, l)$ such that $(p, r l)=1$, and let $\mathcal{F}^{\prime \prime}$ be the remaining contribution.

In the case $p \mid r$, we can apply the Voronoi formula with modulus $r l / p$; estimating the resulting expression directly, one obtains an estimate for the contribution $\mathcal{F}^{\prime \prime}$ to $\mathcal{F}$ that is bounded by

$$
\mathcal{F}^{\prime \prime} \ll \frac{\|\widehat{K}\|_{\infty} Z^{2} X^{3 / 2+\varepsilon}}{q P}
$$

for any $\varepsilon>0$ (see [17, Section 6] for a similar computation, where such contribution is denoted $\left.\mathcal{F}_{1}^{\sharp}\right)$.

Now let $p$ be such that $(p, r l)=1$. By the Voronoi summation formula (Lemma 4.2), we have

$$
\begin{aligned}
& \sum_{n \geqslant 1} \lambda(1, n) e_{r l}(-n p \bar{q}) V_{1}\left(\frac{n}{X}\right) \\
& \quad=(r l)^{3 / 2} \sum_{\sigma \in\{-1,1\}} \sum_{n \geqslant 1} \sum_{m \mid r l} \frac{\lambda(n, m)}{n m^{3 / 2}} \mathrm{Kl}_{2}(\sigma \bar{p} q n ; r l / m) \mathcal{V}_{1, \sigma}\left(\frac{m^{2} n}{r^{3} l^{3} / X}\right) .
\end{aligned}
$$

Therefore, $\mathcal{F}^{\prime}=\mathcal{F}_{1}^{\prime}+\mathcal{F}_{-1}^{\prime}$, where

$$
\mathcal{F}_{\sigma}^{\prime}=\frac{q^{3 / 2} L}{X P|\mathrm{P}||\mathrm{L}|} \sum_{p \in \mathrm{P}} \sum_{l \in \mathrm{L}} \sum_{r \geqslant 1} \widehat{K}(-p \bar{l} \bar{r}) W\left(\frac{r}{R}\right)(r l)^{3 / 2}
$$




$$
\times \sum_{n \geqslant 1} \sum_{m \mid r l} \frac{\lambda(n, m)}{n m^{3 / 2}} \mathrm{Kl}_{2}(\sigma \bar{p} q n ; r l / m) \mathcal{V}_{1, \sigma}\left(\frac{m^{2} n}{r^{3} l^{3} / X}\right) .
$$

We rearrange the sums to get

$$
\begin{aligned}
\mathcal{F}_{\sigma}^{\prime}= & \frac{(q R L)^{3 / 2} L}{X P|\mathrm{P}||\mathrm{L}|} \sum_{r \geqslant 1}\left(\frac{r}{R}\right)^{3 / 2} W\left(\frac{r}{R}\right) \sum_{n, m} \frac{\lambda(n, m)}{\sqrt{n m}} \\
& \times \sum_{p \in \mathrm{P}} \sum_{\substack{l \in \mathrm{L} \\
m \mid r l}} \frac{(l / L)^{3 / 2}}{\sqrt{n} m} \widehat{K}(-p \bar{l} \bar{r}) \mathrm{Kl}_{2}(\sigma \bar{p} q n ; r l / m) \mathcal{V}_{1, \sigma}\left(\frac{m^{2} n}{r^{3} l^{3} / X}\right) .
\end{aligned}
$$

Let $\delta>0$ be a small parameter. For fixed $r$ and $l$, using the bounds from Lemma 4.3 with a suitably large value of $A$, the contribution to the sum over $m$ and $n$ of $(m, n)$ such that

$$
m^{2} n \geqslant q^{\delta} \frac{Z^{3}(r l)^{3}}{X} \asymp \frac{q^{\delta} Z^{3} X^{2} P^{3}}{q^{3}}
$$

is $\ll\|\widehat{K}\|_{\infty} q^{-10}$ (say).

To handle the remaining part of the sum, we apply the Cauchy-Schwarz inequality to the sum over $(m, n)$, and we obtain

$$
\mathcal{F}_{\sigma}^{\prime} \ll \frac{(q R L)^{3 / 2} L}{X P|\mathrm{P}||\mathrm{L}|}\left(\sum_{r \sim R} \sum_{\substack{n, m \geqslant 1 \\ m^{2} n<q^{\delta} Z^{3} X^{2} P^{3} / q^{3}}} \frac{|\lambda(n, m)|^{2}}{n m}\right)^{1 / 2} \mathcal{N}_{\sigma}^{1 / 2}+\|\widehat{K}\|_{\infty} q^{-1},
$$

where

$$
\begin{aligned}
\mathcal{N}_{\sigma}= & \sum_{r, m \geqslant 1} W\left(\frac{r}{R}\right) \frac{1}{m^{2}} \sum_{\substack{p_{1}, p_{2}, l_{1}, l_{2} \\
p_{i} \in \mathrm{P}, l_{i} \in \mathrm{L} \\
m \mid\left(r l_{1}, r l_{2}\right)}}\left(\frac{l_{1} l_{2}}{L^{2}}\right)^{3 / 2} \widehat{K}\left(-p_{1} \bar{l}_{1} \bar{r}\right) \overline{\widehat{K}\left(-p_{2} \bar{l}_{2} \bar{r}\right)} \\
& \times \sum_{n \geqslant 1} \frac{1}{n} \mathrm{Kl}_{2}\left(\sigma \bar{p}_{1} q n ; r l_{1} / m\right) \overline{\mathrm{Kl}_{2}\left(\sigma \bar{p}_{2} q n ; r l_{2} / m\right)} \mathcal{V}_{1, \sigma} \\
& \times\left(\frac{m^{2} n}{r^{3} l_{1}^{3} / X}\right) \overline{\mathcal{V}_{1, \sigma}\left(\frac{m^{2} n}{r^{3} l_{2}^{3} / X}\right)} .
\end{aligned}
$$

We will prove the bound

$$
\mathcal{N}_{\sigma} \ll q^{\varepsilon}\|\widehat{K}\|_{\infty}^{2}\left(Z^{4} R P L+\frac{Z^{3} R^{3 / 2} q^{3} L^{3}}{X^{2} P}\right)
$$


for any $\varepsilon>0$. If we select $\delta>0$ small enough in terms of $\varepsilon$, then by the RankinSelberg bound (4.2), we deduce that

$$
\mathcal{F}_{\sigma}^{\prime} \ll \frac{q^{3 / 2+\varepsilon} Z^{\varepsilon} L^{5 / 2} R^{2}}{X P|\mathrm{P}||\mathrm{L}|} \mathcal{N}_{\sigma}^{1 / 2}+\|\widehat{K}\|_{\infty} q^{-1}
$$

for any $\varepsilon>0$. We conclude, using (6.5) and recalling that $R=X P /(q L)$, that

$$
\begin{aligned}
\mathcal{F}_{\sigma}^{\prime} & \ll \frac{q^{\varepsilon}\|\widehat{K}\|_{\infty} R^{2}(q L)^{3 / 2}}{X P^{2}}\left(Z^{4} R P L+\frac{Z^{3} R^{3 / 2} q^{3} L^{3}}{X^{2} P}\right)^{1 / 2} \\
& \ll q^{\varepsilon}\|\widehat{K}\|_{\infty}\left(\frac{Z^{2} X^{3 / 2} P}{q L^{1 / 2}}+Z^{3 / 2} X^{3 / 4}(q P L)^{1 / 4}\right),
\end{aligned}
$$

for any $\varepsilon>0$. Assuming (6.6), this concludes the proof of Proposition 6.1.

6.1. Proof of (6.6). We will now investigate the inner sum over $n$ in $\mathcal{N}_{\sigma}$, and then perform the remaining summations (over $r, m, p_{i}, l_{i}$ ) essentially trivially. We let

$$
U=\frac{q^{\delta / 2} Z^{3 / 2} X P^{3 / 2}}{q^{3 / 2}}
$$

so that the sum over $m$ has been truncated to $m \leqslant U$.

Let $F$ be a smooth nonnegative function on $\mathbf{R}$ which is supported on $[1 / 2,3]$ and equal to 1 on $[1,2]$. Let $Y \geqslant 1$ be a parameter with

$$
Y \leqslant \frac{q^{\delta} Z^{3} X^{2} P^{3}}{m^{2} q^{3}}
$$

and define

$$
\mathcal{W}_{Y}(x)=\frac{1}{x} \mathcal{V}_{1, \sigma}\left(\frac{m^{2} x Y}{r^{3} l_{1}^{3} / X}\right) \overline{\mathcal{V}_{1, \sigma}\left(\frac{m^{2} x Y}{r^{3} l_{2}^{3} / X}\right)} F(x) .
$$

We study the sums

$$
\mathcal{P}_{Y}=\frac{1}{Y} \sum_{n \geqslant 1} \mathrm{Kl}_{2}\left(\bar{p}_{1} q n ; r l_{1} / m\right) \overline{\mathrm{Kl}_{2}\left(\bar{p}_{2} q n ; r l_{2} / m\right)} \mathcal{W}_{Y}\left(\frac{n}{Y}\right)
$$

and their combinations

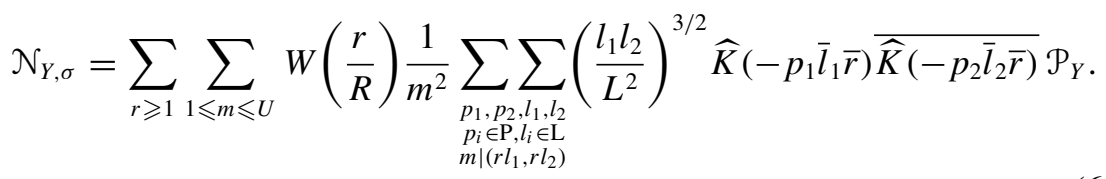


We will prove the following bound: for any $\varepsilon>0$, if $\delta$ is chosen small enough, we have

$$
\mathcal{N}_{Y, \sigma} \ll q^{\varepsilon} Z^{4}\|\widehat{K}\|_{\infty}^{2} R P L+q^{\varepsilon}\|\widehat{K}\|_{\infty}^{2} \frac{Z^{3} R^{3 / 2} q^{3} L^{3}}{X^{2} P} .
$$

Performing a dyadic partition of unity on the $n$ variable in $\mathcal{N}_{\sigma}$, we deduce (6.6).

6.2. Bounding $\mathcal{P}_{\boldsymbol{Y}}$. We apply the Poisson summation formula (1.10) with modulus $r\left[l_{1}, l_{2}\right] / m$ to get

$$
\mathcal{P}_{Y}=\frac{1}{r\left[l_{1}, l_{2}\right] / m} \sum_{n \in \mathbf{Z}} C\left(n, p_{1}, p_{2}, l_{1}, l_{2}, r, m\right) \widehat{\mathcal{W}}_{Y}\left(\frac{n Y}{r\left[l_{1}, l_{2}\right] / m}\right),
$$

where

$$
\begin{aligned}
& C\left(n, p_{1}, p_{2}, l_{1}, l_{2}, r, m\right) \\
& =\sum_{\beta\left(\bmod r\left[l_{1}, l_{2}\right] / m\right)} \mathrm{Kl}_{2}\left(\bar{p}_{1} q \beta ; r l_{1} / m\right) \overline{\mathrm{Kl}}_{2}\left(\bar{p}_{2} q \beta ; r l_{2} / m\right) e_{r\left[l_{1}, l_{2}\right] / m}(\beta n),
\end{aligned}
$$

with $\bar{p}_{i}$ denoting the inverse of $p_{i}$ modulo $r l_{i} / m$. We write

$$
\mathcal{P}_{Y}=\mathcal{P}_{0}+\mathcal{P}_{1},
$$

where

$$
\mathcal{P}_{0}=\frac{1}{r\left[l_{1}, l_{2}\right] / m} C\left(0, p_{1}, p_{2}, l_{1}, l_{2}, r, m\right) \widehat{\mathcal{W}}_{Y}(0)
$$

is the contribution of the term $n=0$ and $\mathcal{P}_{1}$ is the remainder in (6.11). We show below that for any $\varepsilon>0$, if $\delta$ is chosen small enough, we have

$$
\mathcal{P}_{0} \ll \delta_{\substack{l_{1}=l_{2} \\ p_{1}=p_{2}}}(q r)^{\varepsilon} Z^{4}+\delta_{\substack{l_{1}=l_{2} \\ p_{1} \neq p_{2}}} \mathcal{P}_{0} \ll q^{\varepsilon} Z^{4} \frac{m}{r l}\left(\frac{r l}{m}, p_{2}-p_{1}\right)
$$

and that

$$
\mathcal{P}_{1} \ll q^{2 \varepsilon} Z^{3}\left(\frac{r\left[l_{1}, l_{2}\right] 1}{m}\right)^{1 / 2} \frac{m^{2} q^{3}}{X^{2} P^{3}} .
$$

Using (6.12) in the sum (6.9), we find that the contribution to $\mathcal{N}_{Y, \sigma}$ of $\mathcal{P}_{0}$ is bounded by

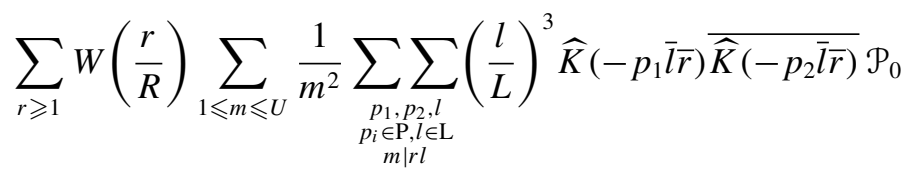




$$
\begin{aligned}
& \ll q^{\varepsilon} Z^{4}\|\widehat{K}\|_{\infty}^{2} \sum_{r \asymp R} \sum_{1 \leqslant m \leqslant U} \frac{1}{m^{2}} \sum_{\substack{l \in \mathrm{L} \\
m \mid r l}}\left(\sum_{p \in \mathrm{P}} 1+\frac{m}{r l} \sum_{\substack{p_{1}, p_{2} \in \mathrm{P} \\
p_{1} \neq p_{2}}}\left(\frac{r l}{m}, p_{2}-p_{1}\right)\right) \\
& \ll q^{\varepsilon} Z^{4}\|\widehat{K}\|_{\infty}^{2}\left(R P L+\sum_{r \asymp R} \frac{1}{r} \sum_{1 \leqslant m \leqslant U} \frac{1}{m} \sum_{\substack{l \in \mathrm{L} \\
m \mid r l}} \frac{1}{l} \sum_{d \mid r l / m} \varphi(d) \sum_{\substack{p_{1}, p_{2} \in \mathrm{P} \\
p_{1} \equiv p_{2}(\bmod d)}} 1\right) \\
& \ll q^{\varepsilon} Z^{4}\|\widehat{K}\|_{\infty}^{2}\left(R P L+P^{2}\right) \ll q^{\varepsilon} Z^{4}\|\widehat{K}\|_{\infty}^{2} R P L .
\end{aligned}
$$

Here $R P L=X P^{2} / q \gg P^{2}$ since $X$ satisfies (6.1). Using (6.13), we find that the contribution of $\mathcal{P}_{1}$ to $\mathcal{N}_{\sigma, Y}$ is bounded by

$$
\begin{aligned}
& \ll q^{\varepsilon} Z^{3}\|\widehat{K}\|_{\infty}^{2} \sum_{r \asymp R} \sum_{1 \leqslant m \leqslant U} \frac{1}{m^{2}} \sum_{\substack{p_{1}, p_{2}, l_{1}, l_{2} \\
m \mid r\left(r l_{1}, r l_{2}\right)}}\left(\frac{r\left[l_{1}, l_{2}\right]}{m}\right)^{1 / 2} \frac{m^{2} q^{3}}{X^{2} P^{3}} \\
& \ll q^{\varepsilon} Z^{3}\|\widehat{K}\|_{\infty}^{2} R \frac{q^{3}}{X^{2} P^{3}}\left(P^{2} L^{2}\right)\left(R L^{2}\right)^{1 / 2} \ll q^{\varepsilon}\|\widehat{K}\|_{\infty}^{2} \frac{Z^{3} R^{3 / 2} q^{3} L^{3}}{X^{2} P} .
\end{aligned}
$$

Combining (6.14) and (6.15), we obtain (6.10).

6.3. Proof of (6.12) and (6.13). The next two lemmas evaluate the archimedean and non-archimedean Fourier transforms which occur in (6.11).

LEMMA 6.2. With notation as above, in particular (6.8), let $j \geqslant 0$ be an integer and let $\varepsilon>0$.

(1) We have

$$
\widehat{\mathcal{W}}_{Y}(0) \ll q^{\delta} Z^{4}
$$

(2) We have

$$
x^{j} \mathcal{W}_{Y}^{(j)}(x) \ll \begin{cases}Z^{2+j}\left(\frac{m^{2} Y q^{3}}{X^{2} P^{3}}\right)^{2-2 \theta_{3}-\varepsilon} & \text { if } Y<\frac{X^{2} P^{3}}{m^{2} q^{3}}, \\ \left(\frac{m^{2} Y q^{3}}{X^{2} P^{3}}\right)^{4 / 3+j / 3} & \text { if } Y \geqslant \frac{X^{2} P^{3}}{m^{2} q^{3}},\end{cases}
$$

where the implied constants depend on $(\varphi, j, \varepsilon)$.

(3) If $1 \leqslant|n| \leqslant q^{\delta} Z \frac{r\left[l_{1}, l_{2}\right]}{m Y}$, then we have

$$
\widehat{\mathcal{W}}_{Y}\left(\frac{n Y}{r\left[l_{1}, l_{2}\right] / m}\right) \ll q^{\delta} Z^{2} \frac{m^{2} Y q^{3}}{X^{2} P^{3}} .
$$


Proof. Since $F_{Y}$ has support in [1/2,3], part (1) follows from $\mathcal{V}_{\sigma}(x) \ll x^{2 / 3}$ for $x \geqslant 1$ and the fact that

$$
\frac{m^{2} x Y}{r^{3} l_{i}^{3} / X} \asymp \frac{m^{2} Y}{X^{2} P^{3} / q^{3}} \ll q^{\delta} Z^{3} .
$$

Part (2) is obtained using the estimates

$$
\begin{array}{cl}
x^{j} \mathcal{V}_{ \pm}^{(j)}(x) \ll Z^{j+1} x^{1-\theta_{3}-\varepsilon} & \text { if } 0<x<1 \\
x^{j} \mathcal{V}_{ \pm}^{(j)}(x) \ll x^{2 / 3+j / 3} & \text { if } x \geqslant 1
\end{array}
$$

(see Lemma 4.3), noting again that $\frac{(r l)^{3}}{X} \sim \frac{X^{2} P^{3}}{q^{3}}$.

From (6.17), for any $n$ such that $1 \leqslant|n| \leqslant q^{\delta} Z \frac{r\left[l_{1}, l_{2}\right]}{m Y}$, we get the estimates

$$
\widehat{\mathcal{W}}_{Y}\left(\frac{n Y}{r\left[l_{1}, l_{2}\right] / m}\right) \ll \begin{cases}Z^{2}\left(\frac{m^{2} Y q^{3}}{X^{2} P^{3}}\right)^{2-2 \theta_{3}-\varepsilon} & \text { if } Y<\frac{X^{2} P^{3}}{m^{2} q^{3}}, \\ \left(\frac{m^{2} Y q^{3}}{X^{2} P^{3}}\right)^{4 / 3} & \text { if } Y \geqslant \frac{X^{2} P^{3}}{m^{2} q^{3}} .\end{cases}
$$

Since $m^{2} Y q^{3} /\left(X^{2} P^{3}\right) \ll q^{\delta} Z^{3}$, the second bound is also

$$
\ll q^{\delta / 3} Z \frac{m^{2} Y q^{3}}{X^{2} P^{3}} .
$$

Together with the first bound, this implies part (3) of the lemma.

LEMMA 6.3. Let $n \in \mathbf{Z}, p_{1}$, $p_{2}$ primes, $l_{1}, l_{2}$ primes, $r \geqslant 1$ and $m \geqslant 1$ be integers with $m \mid r l_{i}$.

(1) We have

$$
C\left(0, p_{1}, p_{2}, l_{1}, l_{2}, r, m\right)=0
$$

unless $l_{1}=l_{2}$.

(2) For l prime with $m \mid r l$, we have

$$
\left|C\left(0, p_{1}, p_{2}, l, l, r, m\right)\right| \leqslant\left(r l / m, p_{2}-p_{1}\right) .
$$

(3) Let

$$
\Delta=q \frac{l_{2}^{2} p_{2}-l_{1}^{2} p_{1}}{\left(l_{1}, l_{2}\right)^{2}}
$$

We have

$$
\left|C\left(n, p_{1}, p_{2}, l_{1}, l_{2}, r, m\right)\right| \leqslant 2^{O(\omega(r))}\left(\frac{r\left[l_{1}, l_{2}\right]}{m}\right)^{1 / 2} \frac{\left(\Delta, n, m / r l_{1}, m / / r l_{2}\right)}{\left(n, m / r l_{1}, m / r l_{2}\right)^{1 / 2}} .
$$


(4) Suppose that $\Delta=0$. If $\left(p_{1}, p_{2}\right)$ are $\equiv 1(\bmod 4)$ and $\left(l_{1}, l_{2}\right)$ are $\equiv 3(\bmod 4)$, then $p_{1}=p_{2}$ and $l_{1}=l_{2}$. For $p$ prime and l prime with $m \mid r l$, we have

$$
|C(n, p, p, l, l, r, m)| \leqslant 2^{O(\omega(r))}\left(\frac{r l}{m}\right)^{1 / 2}\left(n, \frac{r l}{m}\right)^{1 / 2} .
$$

In particular, $C(0, p, p, l, l, r, m) \ll r^{\varepsilon} \frac{r l}{m}$ for any $\varepsilon>0$.

Proof. Part (1) follows by direct computation (the sum vanishes unless $\left[l_{1}, l_{2}\right]=l_{1}$ and $\left[l_{1}, l_{2}\right]=l_{2}$ ). If $n=0$ and $l_{1}=l_{2}$, then

$$
\begin{aligned}
\left|C\left(0, p_{1}, p_{2}, l, l, r, m\right)\right| & =\left|\sum_{\substack{x \bmod r l / m \\
(x, r l / m)=1}} e\left(\frac{\left(p_{2}-p_{1}\right) x}{r l / m}\right)\right| \\
& =\left|\sum_{d \mid\left(r l / m, p_{2}-p_{1}\right)} d \mu\left(\frac{r l}{m d}\right)\right| \leqslant\left(r l / m, p_{2}-p_{1}\right)
\end{aligned}
$$

by a classical bound for Ramanujan's sum, which proves (2). Finally, part (3) is a special case of $\left[12\right.$, Lemma A.2 (A.3)] (applied with $\left(\xi, s_{1}, s_{2}\right)=\left(n, r l_{1} / m\right.$, $\left.r l_{2} / m\right)$, and $\left(a_{1}, b_{1}, a_{2}, b_{2}\right)=\left(q, p_{1}, q, p_{2}\right)$ in the definition of $\left.\Delta\right)$. If $\Delta=0$, then necessarily $p_{1}=p_{2}$ and $l_{1}=l_{2}$, and we obtain (4) immediately.

6.3.1. Estimation of $\mathcal{P}_{0}$. Note that $\mathcal{P}_{0}=0$ unless $l_{1}=l_{2}$. If that is the case, we denote $l=l_{1}=l_{2}$. We then have two bounds for $\mathcal{P}_{0}$. If we have also $p_{1}=p_{2}$, then the quantity $\Delta$ of Lemma 6.3(3) is zero. Since $\widehat{\mathcal{W}}_{Y}(0) \ll q^{\varepsilon} Z^{4}$ for any $\varepsilon>0$ (provided $\delta>0$ is chosen small enough) by Lemma 6.2(1), we obtain

$$
\mathcal{P}_{0} \ll q^{\varepsilon} Z^{4} \frac{m}{r l}\left|C\left(0, p_{1}, p_{1}, l, l, r, m\right)\right| \ll(q r)^{\varepsilon} Z^{4}
$$

by the last part of Lemma 6.3(4).

On the other hand, if $p_{1} \neq p_{2}$, we have $\Delta \neq 0$; hence,

$$
\mathcal{P}_{0} \ll q^{\varepsilon} Z^{4} \frac{m}{r l}\left(\frac{r l}{m}, p_{2}-p_{1}\right)
$$

by Lemma 6.3(1) (which shows that the sum $C\left(0, p_{1}, p_{2}, l_{1}, l_{2}, r, m\right)$ is zero unless $l_{1}=l_{2}$ ) and (2).

6.3.2. Estimation of $\mathcal{P}_{1}$. Using Lemma 6.2(2) for a suitable value of $j$, we obtain first

$\mathcal{P}_{1}=\frac{1}{r\left[l_{1}, l_{2}\right] / m} \sum_{1 \leqslant|n| \leqslant q^{\delta} Z \frac{r\left[l_{1}, l_{2}\right]}{m Y}} C\left(n, p_{1}, p_{2}, l_{1}, l_{2}, r, m\right) \widehat{\mathcal{W}}_{Y}\left(\frac{n Y}{r\left[l_{1}, l_{2}\right] / m}\right)+O\left(q^{-1}\right)$ 
for any $\varepsilon>0$ if $\delta$ is chosen small enough. Then, by Lemmas 6.3 and 6.2(3), we deduce that

$$
\begin{aligned}
\mathcal{P}_{1} & \ll q^{\varepsilon+2 \delta} \frac{1}{\left(r\left[l_{1}, l_{2}\right] / m\right)^{1 / 2}} \sum_{1 \leqslant|n| \leqslant q^{\delta} Z \frac{r\left[l_{1}, l_{2}\right]}{m Y}} \frac{\left(\Delta, n, r l_{1} / m, r l_{2} / m\right)}{\left(n, r l_{1} / m, r l_{2} / m\right)^{1 / 2}} \frac{Z^{2} m^{2} Y q^{3}}{X^{2} P^{3}} \\
& \ll q^{2 \varepsilon} Z^{3}\left(\frac{r\left[l_{1}, l_{2}\right] 1}{m}\right)^{1 / 2} \frac{m^{2} q^{3}}{X^{2} P^{3}}
\end{aligned}
$$

if $\delta<\varepsilon / 2$.

\section{Estimate of $\mathcal{O}$}

In this section, we bound the sum $\mathcal{O}$ defined in (5.4). Our goal is the following.

Proposition 7.1. Let $\eta>0$ be a parameter such that (6.1) holds. Let $\varepsilon>0$. If $\delta$ is a sufficiently small positive real number and if $P, L, X$ satisfy

$$
X P \leqslant q^{2} L, \quad q^{1+\delta} L^{2}<X / 8, \quad q^{\delta} P H L<q / 8,
$$

then we have

$$
\mathcal{O} \ll q^{\varepsilon}\|\widehat{K}\|_{\infty} \frac{q X^{1 / 2}}{P},
$$

where the implied constant depends on $\varphi$ and $\varepsilon$.

We start by decomposing $\mathcal{O}$ into

$$
\mathcal{O}=\mathcal{O}_{1}+\mathcal{O}_{2}
$$

according to whether the prime $l$ divides $h$ or not; in other words,

$$
\begin{aligned}
\mathcal{O}_{1} & =\frac{1}{|\mathrm{P}||\mathrm{L}|} \sum_{p \in \mathrm{P}} \sum_{l \in \mathrm{L}} \sum_{h \neq 0} \widehat{W}\left(\frac{h l}{H}\right) \sum_{n \geqslant 1} \lambda(1, n) K(n, h p) V\left(\frac{n}{X}\right) \\
\mathcal{O}_{2} & =\frac{1}{|\mathrm{P}||\mathrm{L}|} \sum_{p \in \mathrm{P}} \sum_{l \in \mathrm{L}} \sum_{\substack{h \neq 0 \\
(h, l)=1}} \widehat{W}\left(\frac{h}{H}\right) \sum_{n \geqslant 1} \lambda(1, n) K(n, h p \bar{l}) V\left(\frac{n}{X}\right) .
\end{aligned}
$$

Both of these sums will be handled in a similar way in the next two subsections, beginning with the most difficult one. 
7.1. Bounding $\mathcal{O}_{2}$. In the sum $\mathcal{O}_{2}$, we first use the bound

$$
\widehat{W}(x) \ll(1+|x|)^{-A}
$$

for any $A \geqslant 1$ and $x \in \mathbf{R}$, and

$$
\sum_{n \geqslant 1} \lambda(1, n) K(n, h p \bar{l}) V\left(\frac{n}{X}\right) \ll X^{1+\varepsilon} q^{1 / 2}\|\widehat{K}\|_{\infty} \ll q^{5 / 2+2 \varepsilon}\|\widehat{K}\|_{\infty}
$$

for any $\varepsilon>0$ (by (4.1) and discrete Fourier inversion) to truncate the sum over $h$ to $|h| \leqslant q^{\delta} H$, for some $\delta>0$ that may be arbitrarily small.

Let $T \geqslant 0$ be a smooth function with compact support such that $T(x)=\|V\|_{\infty}$ for $x \in[1 / 2,3]$ and such that $T$ satisfies (1.5) with a fixed value of $Z$. We then have $|V| \leqslant T$.

In the sum $\mathrm{O}_{2}$, we split the $h$-sum into $O(\log q)$ dyadic sums. We then apply the Cauchy-Schwarz inequality to smooth the $n$-variable, and we obtain

$$
\mathcal{O}_{2} \ll \frac{\log ^{3} q}{P L}\left(\sum_{n \sim X}|\lambda(1, n)|^{2}\right)^{1 / 2} \max _{1 \leqslant H^{\prime} \leqslant q^{\delta} H} \mathcal{R}_{H^{\prime}}^{1 / 2} \ll \frac{X^{1 / 2} \log ^{3} q}{P L} \max _{1 \leqslant H^{\prime} \leqslant q^{\delta} H} \mathcal{R}_{H^{\prime}}^{1 / 2},
$$

by (4.1) again, where

$$
\mathcal{R}_{H^{\prime}}=\sum_{p_{1}, h_{1}, l_{1}, p_{2}, h_{2}, l_{2}} \sum_{n \geqslant 1} K\left(n, h_{1} p_{1} \bar{l}_{1}\right) \overline{K\left(n, h_{2} p_{2} \bar{l}_{2}\right)} \widehat{W}\left(\frac{h_{1}}{H}\right) \overline{\widehat{W}\left(\frac{h_{2}}{H}\right)} T\left(\frac{n}{X}\right),
$$

with the variables in the sums constrained by the conditions

$$
p_{i} \in \mathrm{P}, \quad l_{i} \in \mathrm{L}, \quad H^{\prime}<h_{i} \leqslant 2 H^{\prime}, \quad\left(l_{i}, h_{i}\right)=1 .
$$

For $x \in \mathbf{F}_{q}$, we define

$$
v(x)=\sum_{\substack{(p, h, l) \in \mathrm{P} \times\left[H^{\prime}, 2 H^{\prime}[\times \mathrm{L},(h, l)=1 \\ p h \bar{l} \equiv x(\bmod q)\right.}} \widehat{W}\left(\frac{h}{H}\right)
$$

so that we have

$$
\mathcal{R}_{H^{\prime}}=\sum_{x_{1}, x_{2} \in \mathbf{F}_{q}} v\left(x_{1}\right) v\left(x_{2}\right) \sum_{n \geqslant 1} K\left(n, x_{1}\right) \overline{K\left(n, x_{2}\right)} T\left(\frac{n}{X}\right) .
$$

We apply the Poisson summation formula (1.10) for the sum over $n$. This results in the formula

$$
\sum_{n \geqslant 1} K\left(n, x_{1}\right) \overline{K\left(n, x_{2}\right)} T\left(\frac{n}{X}\right)
$$




$$
=\frac{X}{\sqrt{q}} \sum_{h \in \mathbf{Z}}\left(\frac{1}{\sqrt{q}} \sum_{n(\bmod q)} K\left(n, x_{1}\right) \overline{K\left(n, x_{2}\right)} e\left(\frac{n h}{q}\right)\right) \widehat{T}\left(\frac{h X}{q}\right) .
$$

Observe that for any $h \in \mathbf{Z}$, we have

$$
\frac{1}{\sqrt{q}} \sum_{n(\bmod q)} K\left(n, x_{1}\right) \overline{K\left(n, x_{2}\right)} e\left(\frac{n h}{q}\right)=\frac{1}{\sqrt{q}} \sum_{u(\bmod q)} \widehat{K}\left(u, x_{1}\right) \widehat{\widehat{K}}\left(u+h, x_{2}\right)
$$

where $\widehat{K}(u, x)$ is defined as in (2.1). In particular, this quantity is bounded by $q^{1 / 2}\|\widehat{K}\|_{\infty}^{2}$.

Now, for all $h \neq 0$ and all $A \geqslant 1$, we have

$$
\widehat{T}\left(\frac{h X}{q}\right) \ll_{A}\left(\frac{q Z}{h X}\right)^{A} \leqslant\left(\frac{q Z}{X}\right)^{A} \leqslant q^{-A \eta},
$$

by (6.1), where the implied constant depends on $A$. Hence, taking $A$ large enough in terms of $\eta$, the contribution of all $h \neq 0$ to the sum over $n$ is $\ll\|\widehat{K}\|_{\infty}^{2} q^{-5}$, and the total contribution to $\mathcal{R}_{H^{\prime}}$ is (using very weak bounds on $v(x)$ )

$$
\ll\|\widehat{K}\|_{\infty}^{2} q^{-3}(P H L)^{2} \ll\|\widehat{K}\|_{\infty}^{2} q^{-1}
$$

by (7.1).

The remaining contribution to $\mathcal{R}_{H^{\prime}}$ from the frequency $h=0$ is

$$
\frac{X}{\sqrt{q}} \sum_{x_{1}, x_{2} \in \mathbf{F}_{q}} \sum v\left(x_{1}\right) v\left(x_{2}\right) \frac{1}{\sqrt{q}} \sum_{u \in \mathbf{F}_{q}} \widehat{K}\left(u, x_{1}\right) \widehat{\widehat{K}}\left(u, x_{2}\right) \widehat{T}(0) .
$$

LEMMA 7.2. For any $\left(x_{1}, x_{2}\right) \in \mathbf{F}_{q} \times \mathbf{F}_{q}$, we have

$$
\frac{1}{\sqrt{q}} \sum_{u \in \mathbf{F}_{q}} \widehat{K}\left(u, x_{1}\right) \overline{\widehat{K}}\left(u, x_{2}\right)=L\left(x_{1}-x_{2}\right)
$$

where

$$
L(x)=\frac{1}{\sqrt{q}} \sum_{u \in \mathbf{F}_{q}^{\times}}|\widehat{K}(u)|^{2} e_{q}(-\bar{u} x)+\frac{1}{\sqrt{q}}|\widehat{K}(0)|^{2} .
$$

Moreover, we have

$$
\widehat{L}(h)=|\widehat{K}(0)|^{2} \delta_{h \equiv 0(\bmod q)}+|\widehat{K}(\bar{h})|^{2} \delta_{h \equiv 0(\bmod q)},
$$

and, in particular, $|\widehat{L}(h)| \leqslant\|\widehat{K}\|_{\infty}^{2}$ for all $h \in \mathbf{F}_{q}$. 
Proof. The first formula is an immediate consequence of the definition (2.1), and the second results from a straightforward computation.

LEMMA 7.3. We have

$$
\|v\|_{2}^{2}=\sum_{x \in \mathbf{F}_{q}} v(x)^{2} \ll q^{\varepsilon+\delta} P H L
$$

for any $\varepsilon>0$.

Proof. From the last condition in (7.1), we have the implications

$$
h_{2} p_{2} \bar{l}_{2}=h_{1} p_{1} \bar{l}_{1}(\bmod q) \Longleftrightarrow l_{1} h_{2} p_{2} \equiv l_{2} h_{1} p_{1}(\bmod q) \Longleftrightarrow l_{1} h_{2} p_{2}=l_{2} h_{1} p_{1} .
$$

Therefore, if $\left(p_{1}, h_{1}, l_{2}\right)$ are given, the number of possibilities for $\left(p_{2}, h_{2}, l_{1}\right)$ is $\ll q^{\varepsilon}$ for any $\varepsilon>0$. The bound

$$
\sum_{x \in \mathbf{F}_{q}^{\times}} v(x)^{2} \ll q^{\varepsilon} P H^{\prime} L \leqslant q^{\varepsilon+\delta} P H L
$$

follows immediately.

We can now combine these two lemmas with Proposition 4.1 to deduce that

$$
\begin{aligned}
\frac{X}{\sqrt{q}}\left|\sum_{x_{1}, x_{2} \in \mathbf{F}_{q}} \sum v\left(x_{1}\right) v\left(x_{2}\right) \frac{1}{\sqrt{q}} \sum_{u \in \mathbf{F}_{q}} \widehat{K}\left(u, x_{1}\right) \overline{\widehat{K}\left(u, x_{2}\right)} \widehat{T}(0)\right| & \leqslant X\|\widehat{L}\|_{\infty}\|v\|_{2}^{2}|\widehat{T}(0)| \\
& \ll q^{\varepsilon}\|\widehat{K}\|_{\infty}^{2} X P H L
\end{aligned}
$$

for any $\varepsilon>0$, by taking $\delta$ small enough in terms of $\varepsilon$. Hence, we obtain

$$
\mathcal{O}_{2} \ll q^{\varepsilon}\|\widehat{K}\|_{\infty} X\left(\frac{H}{P L}\right)^{1 / 2} \ll q^{1+\varepsilon}\|\widehat{K}\|_{\infty} \frac{X^{1 / 2}}{P} .
$$

7.2. Bounding $\mathcal{O}_{1}$ and the end of the proof of Proposition 7.1. The treatment of $\mathcal{O}_{1}$ is similar to that of $\mathcal{O}_{2}$ but simpler, and so we will be brief. We have

$$
\mathcal{O}_{1}=\frac{1}{|\mathrm{~L}|} \sum_{l \in \mathrm{L}} \frac{1}{|\mathrm{P}|} \sum_{p \in \mathrm{P}} \sum_{h \neq 0} \widehat{W}\left(\frac{h}{H / l}\right) \sum_{n \geqslant 1} \lambda(1, n) K(n, h p) V\left(\frac{n}{X}\right) .
$$

We bound the sum over $p$ for each individual $l \in \mathrm{L}$, with $h \ll H / l \asymp H / L$, by repeating the arguments of the previous section with $H$ replaced by $H / l$ and $L$ 
replaced by 1 . We obtain

$$
\mathcal{O}_{1} \ll\|\widehat{K}\|_{\infty} q^{\varepsilon} X\left(\frac{H}{P L}\right)^{1 / 2} \ll q^{1+\varepsilon}\|\widehat{K}\|_{\infty} \frac{X^{1 / 2}}{P}
$$

for any $\varepsilon>0$, as in the previous case.

Finally, since $\mathcal{O}=\mathcal{O}_{1}+\mathcal{O}_{2}$, this bound combined with (7.6) implies Proposition 7.1.

\section{End of the proof}

We can now finish the proof of our main theorem. We recall that $X, Z$ are such that

$$
Z^{2 / 3} q^{4 / 3} \leqslant X \leqslant Z^{-2} q^{2}
$$

In particular, $Z \leqslant q^{1 / 4}$ and

$$
X \geqslant Z^{2 / 3} q^{4 / 3} \geqslant Z q^{1+1 / 4}
$$

therefore, (6.1) holds for $\eta=1 / 4$.

Assuming that the conditions (7.1) hold and combining (5.3), Proposition 6.1 and Proposition 7.1, we deduce the estimate

$$
S_{V}(K, X) \ll q^{\varepsilon}\|\widehat{K}\|_{\infty}\left(\frac{Z^{2} X^{3 / 2} P}{q L^{1 / 2}}+Z^{3 / 2} X^{3 / 4}(q P L)^{1 / 4}+\frac{q X^{1 / 2}}{P}\right)
$$

for any $\varepsilon>0$. When $L=Z^{2 / 3} X P / q^{5 / 3}$, the first two terms are equal to $Z^{5 / 3} X P^{1 / 2} / q^{1 / 6}$. For $P=q^{7 / 9} /\left(X^{1 / 3} Z^{10 / 9}\right)$, they are also equal to the third term which is $Z^{10 / 9} q^{2 / 9} X^{5 / 6}$. Moreover, the conditions (8.1) and $Z \leqslant q^{1 / 4}$ imply then by simple computations that

$$
1 \leqslant P, \quad 1 \leqslant L, \quad L \leqslant P^{4}, \quad X P \leqslant q^{2} L
$$

(for instance, $X^{3} Z^{10} \leqslant Z^{10}\left(q^{2} / Z^{2}\right)^{3}=Z^{4} q^{6} \leqslant q^{7}$ gives $P \geqslant 1$ ), and then we get

$$
q^{1+\delta} L^{2}<\frac{X}{8}
$$

for $\delta=1 / 18$, provided $q$ is large enough (since $q L^{2}=q^{-7 / 9} Z^{-8 / 9} X^{4 / 3} \leqslant$ $X\left(X^{1 / 3} q^{-7 / 9}\right) \leqslant X q^{-1 / 9}$ using $\left.X \leqslant q^{2}\right)$. By (5.1), this also implies that $q^{\delta} P H L<$ $q / 8$. Hence, this choice of the parameters satisfies (7.1). We finally conclude that

$$
S_{V}(K, X) \ll\|\widehat{K}\|_{\infty} Z^{10 / 9} q^{2 / 9+\varepsilon} X^{5 / 6}
$$

for any $\varepsilon>0$. 


\section{Applications}

In this section, we prove Corollaries 1.4 and 1.5.

9.1. Proof of Corollary 1.4. Applying the approximate functional equation for $L(\varphi \otimes \chi, s)$ in the balanced form, we immediately express the first moment

$$
\frac{1}{q-1} \sum_{\chi(\bmod q)} M(\chi) L(\varphi \otimes \chi, 1 / 2)
$$

in terms of the sums

$$
\frac{1}{\sqrt{q}} \sum_{n \geqslant 1} \frac{\lambda(1, n)}{\sqrt{n}} K(n) V\left(\frac{n}{q^{3 / 2}}\right)
$$

and

$$
\frac{1}{\sqrt{q}} \sum_{n \geqslant 1} \frac{\overline{\lambda(1, n)}}{\sqrt{n}} L(n) V\left(\frac{n}{q^{3 / 2}}\right),
$$

for suitable test functions satisfying (1.5) for $Z=1$, where

$$
K(n)=\frac{q^{1 / 2}}{q-1} \sum_{\chi(\bmod q)} M(\chi) \chi(n), \quad L(n)=\frac{q^{1 / 2}}{q-1} \sum_{\chi(\bmod q)} \tau(\chi)^{3} M(\chi) \overline{\chi(n)},
$$

in terms of the normalized Gauss sum $\tau(\chi)$. An elementary computation shows that this function $L$ coincides with the function in the statement of Corollary 1.4. Since, moreover, the $\overline{\lambda(1, n)}$ are the Hecke eigenvalues of the dual cuspform $\tilde{\varphi}$, the corollary follows from Theorem 1.3 applied to $K$ and $L$.

REMARK 10. (1) If

$$
M(\chi)=\frac{1}{\sqrt{q}} \sum_{x \in \mathbf{F}_{q}^{\times}} K(x) \overline{\chi(x)}
$$

is the discrete Mellin transform of the trace function $K$ of a Fourier sheaf $\mathcal{F}$ that is a middle-extension sheaf on $\mathbf{G}_{m}$ of weight 0 and if no sheaf of the form $\left[x \mapsto x^{-1}\right]^{*} \mathrm{D}\left(\mathcal{K} \ell_{3}\right)$ is among the geometrically irreducible components of $\mathcal{F}$, then both $\|\widehat{K}\|_{\infty}$ and $\|\widehat{L}\|_{\infty}$ are bounded in terms of the conductor of $\mathcal{F}$ only, and we obtain

$$
\frac{1}{q-1} \sum_{\chi(\bmod q)} M(\chi) L(\varphi \otimes \chi, 1 / 2) \ll q^{2 / 9+\varepsilon}
$$

for any $\varepsilon>0$, where the implied constant depends only on $\varepsilon, \varphi$ and the conductor of $\mathcal{F}$. 
(2) Applying the approximate functional equation in a balanced form may not always be the best move. For instance, consider the important special case where $M(\chi)=1$. We are then evaluating the first moment

$$
\frac{1}{q-1} \sum_{\chi(\bmod q)} L(\varphi \otimes \chi, 1 / 2)
$$

of the central values of the twisted $L$-functions. Then we are working with the functions

$$
K(n)=q^{1 / 2} \delta_{n \equiv 1(\bmod q)}, \quad L(n)=\mathrm{Kl}_{3}(n ; q),
$$

whose Fourier transforms are bounded by absolute constants. Hence, the above leads to

$$
\frac{1}{q-1} \sum_{\chi(\bmod q)} L(\varphi \otimes \chi, 1 / 2) \ll q^{2 / 9+\varepsilon}
$$

for any $\varepsilon>0$, where the implied constant depends on $\varphi$ and $\varepsilon$.

On the other hand, the approximate functional equation in the unbalanced form yields sums of the shape

$$
\sum_{n \equiv 1(\bmod q)} \frac{\lambda(1, n)}{\sqrt{n}} V\left(\frac{n}{Y q^{3 / 2}}\right) \text { and } \frac{1}{\sqrt{q}} \sum_{n \geqslant 1} \frac{\overline{\lambda(1, n)}}{\sqrt{n}} \mathrm{Kl}_{3}(n ; q) V\left(\frac{n Y}{q^{3 / 2}}\right),
$$

for some parameter $Y>0$ at our disposal. Assuming the Ramanujan-Petersson conjecture for $\varphi$ and $\widetilde{\varphi}$ and using Deligne's bound $\left|\mathrm{Kl}_{3}(n ; q)\right| \leqslant 3$ for $(n, q)=1$, we obtain the much stronger bound

$$
\frac{1}{q-1} \sum_{\chi(\bmod q)} L(\varphi \otimes \chi, 1 / 2)=1+(q Y)^{\varepsilon}\left(Y^{1 / 2} / q^{1 / 4}+q^{1 / 4} / Y^{1 / 2}\right) \ll q^{\varepsilon}
$$

for any $\varepsilon>0$, on choosing $Y=q^{1 / 2}$.

Note that, again under the Ramanujan-Petersson conjecture for $\varphi$ and its dual, we would obtain an asymptotic formula for the first moment (9.1), provided we could obtain an estimate for $S_{V}\left(\mathrm{Kl}_{3}, X\right)$ with a power-saving in terms of $q$, when $X$ is a bit smaller than $q$. Results of this type are however currently only known if $\varphi$ is an Eisenstein series (starting from the work [9] of Friedlander and Iwaniec for the ternary divisor function; see also the papers of Fouvry et al. [7], Kowalski et al. [16] and Zacharias [24]).

This illustrates the importance of the problem of obtaining nontrivial bounds for short sums in Theorem 1.3. However, we expect that much more refined properties of trace functions and their associated sheaves will be necessary for such a purpose (as indicated by Remark 5). 
9.2. Proof of Corollary 1.5. The symmetric square $\varphi$ of $\psi$ has Hecke eigenvalues

$$
\lambda(1, n)=\sum_{d^{2} \mid n} \lambda\left(\frac{n^{2}}{d^{2}}\right)
$$

and, hence, by Möbius inversion, we have

$$
\lambda\left(n^{2}\right)=\sum_{d^{2} \mid n} \mu(d) \lambda\left(1, \frac{n}{d^{2}}\right) .
$$

We deduce that

$$
\sum_{n \geqslant 1} \lambda\left(n^{2}\right) K(n) V\left(\frac{n}{X}\right)=\sum_{d \geqslant 1} \mu(d) \sum_{n \geqslant 1} K\left(n d^{2}\right) \lambda(1, n) V\left(\frac{n d^{2}}{X}\right) .
$$

For

$$
1 \leqslant d \leqslant \frac{X^{1 / 2}}{Z^{1 / 3} q^{2 / 3}}
$$

we can apply Theorem 1.3 to the sum over $n$ and the $q$-periodic function $L(n)=$ $K\left(n d^{2}\right)$, with $X$ replaced by $X / d^{2}$. Since $q \nmid d$, we have $\widehat{L}(x)=\widehat{K}\left(\bar{d}^{2} x\right)$ for any $x \in \mathbf{Z}$ so that $\|\widehat{L}\|_{\infty}=\|\widehat{K}\|_{\infty}$, and we get

$$
\begin{aligned}
\sum_{d \leqslant X^{1 / 2} /\left(Z^{1 / 3} q^{2 / 3}\right)} \mu(d) \sum_{n \geqslant 1} K\left(n d^{2}\right) \lambda(1, n) V\left(\frac{n d^{2}}{X}\right) & \ll\|\widehat{K}\|_{\infty} Z^{10 / 9} q^{2 / 9+\varepsilon} \sum_{d \geqslant 1} \frac{X^{5 / 6}}{d^{5 / 3}} \\
& \ll\|\widehat{K}\|_{\infty} Z^{10 / 9} q^{2 / 9+\varepsilon} X^{5 / 6}
\end{aligned}
$$

for any $\varepsilon>0$.

Since $V$ has compact support in [1/2,3], the sum over $n$ is empty if $d \geqslant \sqrt{3 X}$. Since

$$
\sum_{n \geqslant 1} K\left(n d^{2}\right) \lambda(1, n) V\left(\frac{n d^{2}}{X}\right) \ll\|K\|_{\infty}\left(\frac{X}{d^{2}}\right)^{1+\varepsilon}
$$

for any $\varepsilon>0$, by the Rankin-Selberg bound (4.1), we can estimate the remaining part of the sum as follows:

$$
\begin{gathered}
\sum_{X^{1 / 2} /\left(Z^{1 / 3} q^{2 / 3}\right)<d \leqslant \sqrt{3 X}} \mu(d) \sum_{n \geqslant 1} K\left(n d^{2}\right) \lambda(1, n) V\left(\frac{n d^{2}}{X}\right) \\
\ll\|K\|_{\infty} X^{1+\varepsilon} \sum_{X^{1 / 2} /\left(Z^{1 / 3} q^{2 / 3}\right)<d \leqslant \sqrt{3 X}} \frac{1}{d^{2+2 \varepsilon}} \ll\|K\|_{\infty} X^{1 / 2+\varepsilon} Z^{1 / 3} q^{2 / 3}
\end{gathered}
$$

for any $\varepsilon>0$. 
REMARK 11. The additional dependency on $\|K\|_{\infty}$ seems to be unavoidable in Corollary 1.5.

\section{Proof of Corollary 1.6}

The proof of Corollary 1.6 requires additional ingredients besides Theorem 1.3. We will be somewhat brief in handling these additional arguments (especially standard analytic arguments) since similar computations have been performed in a number of other papers (for example [4]).

First, in terms of the Hecke eigenvalues $\lambda(m, n)$ of the symmetric square $\psi$ of $\varphi$, we have the identity

$$
\lambda(n)^{2}=\sum_{d^{2} b c=n} \mu(d) \lambda(1, c)
$$

(see (9.2) and (1.9)). Thus, we have

$$
\sum_{n \geqslant 1} \lambda(n)^{2} K(n) V\left(\frac{n}{X}\right)=\sum_{d, m, n \geqslant 1} \mu(d) \lambda(1, n) K\left(d^{2} m n\right) V\left(\frac{d^{2} m n}{X}\right) .
$$

We bound the contribution of the integers $n$ divisible by $q$ using the KimSarnak bound [15] for $\lambda(1, n)$, which shows that it is

$$
\ll\|K\|_{\infty} X^{1+\varepsilon} q^{-1+7 / 32},
$$

for any $\varepsilon>0$, and hence is negligible. We may therefore restrict the sum on the right-hand side to integers such that $(d m n, q)=1$.

For a fixed value of $d \leqslant D$, coprime to $q$, we consider the sum

$$
T_{d, x}=\sum_{m, n \geqslant 1} \lambda(1, n) K\left(d^{2} m n\right) V\left(\frac{d^{2} m n}{X}\right) .
$$

We need to estimate the sum of $T_{d, x}$ over $d \geqslant 1$.

Let $D \geqslant 1$ be some small parameter to be fixed later. The contribution of the integers $d>D$ is bounded trivially and is

$$
\sum_{d>D} T_{d, X} \ll \frac{\|K\|_{\infty} X^{1+\varepsilon}}{D}
$$

for any $\varepsilon>0$. 
We now fix $d \leqslant D$, coprime to $q$. We handle the sum $T_{d, X}$ by a smooth dyadic partition of unity on the $m$-variable. This reduces the problem to estimates of $O(\log X)$ sums of the form

$$
S_{d, M}=\sum_{\substack{m, n \geqslant 1 \\(m n, q)=1}} \lambda(1, n) K\left(d^{2} m n\right) V\left(\frac{d^{2} m n}{X}\right) W\left(\frac{m}{M}\right),
$$

where $W$ is smooth and compactly supported in $[1 / 2,5 / 2]$. We set

$$
N=\frac{X}{d^{2} M}
$$

so that $n \sim N$ in the sum.

The estimate for (10.2) splits in three cases, depending on the size of $M$.

10.1. When $M$ is small. We assume that $\frac{X}{d^{2} m} \geqslant \frac{X}{D^{2} M} \geqslant Z^{2 / 3} q^{4 / 3}$ or, in other words, that

$$
D^{2} M \leqslant \frac{X}{Z^{2 / 3} q^{4 / 3}} .
$$

We can then apply Theorem 1.3, and we derive

$$
\begin{aligned}
S_{d, M} & \ll\|K\|_{\infty} q^{7 / 32-1} \frac{X^{1+\varepsilon}}{d^{2}}+\|\widehat{K}\|_{\infty} Z^{10 / 9} q^{2 / 9+\varepsilon} \sum_{m \sim M}\left(\frac{X}{d^{2} m}\right)^{5 / 6} \\
& \ll\|K\|_{\infty} X^{\varepsilon} q^{7 / 32-1} \frac{X}{d^{2}}+\|\widehat{K}\|_{\infty} X^{\varepsilon} Z^{10 / 9}\left(\frac{X}{d^{2}}\right)^{5 / 6} q^{2 / 9} M^{1 / 6}
\end{aligned}
$$

for any $\varepsilon>0$ (the first term corresponds to removing the constraint $(n, q)=1$ ).

10.2. When $M$ is in the Fourier range. If $M \geqslant q^{1 / 2}$, then it is beneficial to apply the Poisson summation formula to the $m$-variable. As in the previous case, the cost of removing the condition $(m, q)=1$ is $\ll\|K\|_{\infty} q^{7 / 32-1} X^{1+\varepsilon} / d^{2}$ for $\varepsilon>0$. The Poisson summation formula implies that

$$
\sum_{m \geqslant 1} K\left(d^{2} m n\right) V\left(\frac{d^{2} m n}{X}\right) W\left(\frac{m}{M}\right) \ll\|\widehat{K}\|_{\infty}\left(\frac{M}{q^{1 / 2}}+q^{1 / 2}\right),
$$

and, therefore, (10.2) is bounded by

$$
S_{d, M} \ll\|K\|_{\infty} X^{\varepsilon} q^{7 / 32-1} \frac{X}{d^{2}}+\|\widehat{K}\|_{\infty} X^{\varepsilon} \frac{X}{d^{2}}\left(\frac{1}{q^{1 / 2}}+\frac{q^{1 / 2}}{M}\right)
$$

for any $\varepsilon>0$. 
10.3. When $M$ is large but not in Fourier range. If $M \leqslant q^{1 / 2}$, thinking of the prototypical case when $X \sim q^{3 / 2}$ and $D$ is close to one, the $n$-sum is of length close to $q$, so the natural move is to smooth the $n$-sum and then use the Poisson summation formula on the resulting sums.

Thus, we apply the Cauchy-Schwarz inequality to (10.2), leaving the $n$ variable outside, namely

$$
\begin{aligned}
& \left|S_{d, M}\right|^{2} \ll \sum_{n \sim X / d^{2} M}|\lambda(1, n)|^{2} \\
& \times \sum_{\substack{m_{i} \sim M \\
\left(m_{i}, q\right)=1}} \sum_{n \geqslant 1} K\left(d^{2} m_{1} n\right) \overline{K\left(d^{2} m_{2} n\right)} V\left(\frac{d^{2} m_{1} n}{X}\right) \bar{V}\left(\frac{d^{2} m_{2} n}{X}\right) .
\end{aligned}
$$

Here, we have dropped the constraint $(n, q)=1$ on the right-hand side by positivity and replaced the expressions $W\left(\frac{m_{i}}{M}\right)$ by the summation conditions $m_{i} \sim M$.

By the Poisson summation formula, we have

$$
\begin{aligned}
& \sum_{n \geqslant 1} K\left(d^{2} m_{1} n\right) \overline{K\left(d^{2} m_{2} n\right)} V\left(\frac{d^{2} m_{1} n}{X}\right) \bar{V}\left(\frac{d^{2} m_{2} n}{X}\right) \\
& =\frac{N}{q^{1 / 2}} \sum_{h \in \mathbf{Z}} \widehat{K}_{(2)}(h) \mathcal{W}\left(\frac{h}{q /\left(X / d^{2} M\right)}\right),
\end{aligned}
$$

where $\mathcal{W}(y)$ is a smooth function depending on $d, m_{1}, m_{2}$, rapidly decaying as $y \rightarrow \infty$, and

$$
\widehat{K}_{(2)}(h)=\frac{1}{\sqrt{q}} \sum_{n \in \mathbf{F}_{q}} K\left(d^{2} m_{1} n\right) \overline{K\left(d^{2} m_{2} n\right)} e\left(\frac{n h}{q}\right) .
$$

To go further, we use the assumption of Corollary 1.6 that $K$ is the trace function of a middle-extension $\ell$-adic sheaf $\mathcal{F}$ that is not exceptional. Indeed, from [4, Theorem 6.3], we can deduce that there exists a set $B \subset \mathbf{F}_{q}^{\times}$such that $|B|$ is bounded in terms of the conductor of $\mathcal{F}$ only and such that whenever

$$
m_{1} / m_{2}(\bmod q) \notin B
$$

then we have

$$
\left\|\widehat{K}_{(2)}\right\|_{\infty} \ll 1
$$

where the implied constant depends on the conductor of $\mathcal{F}$ only. 
Returning to (10.6), we apply the bound (10.7) to the pairs $\left(m_{1}, m_{2}\right)$ which satisfy (10.8) and apply the trivial bound otherwise.

We see then that the contribution to the second factor of (10.6) of the 'diagonal' pairs not satisfying (10.8) is bounded by

$$
\ll X^{\varepsilon} M\left(\frac{M}{q}+1\right) \frac{X / M}{d^{2}}
$$

for any $\varepsilon>0$, while the contribution of the pairs $\left(m_{1}, m_{2}\right)$ satisfying (10.8) is bounded by

$$
\ll X^{\varepsilon} M^{2}\left(\frac{X / M}{d^{2} q^{1 / 2}}+q^{1 / 2}\right),
$$

for any $\varepsilon>0$, where in both cases the implied constant depends only on $\varepsilon$ and on the conductor of $\mathcal{F}$.

Collecting these bounds, we obtain from (10.6) the bound

$$
S_{d, M} \ll \frac{X^{1+\varepsilon}}{d^{2}}\left(\frac{1}{M^{1 / 2}}+\frac{1}{q^{1 / 4}}+q^{1 / 4} M^{1 / 2} \frac{d}{X^{1 / 2}}\right),
$$

for any $\varepsilon>0$, where the implied constant depends only on $\varepsilon$ and on the conductor of $\mathcal{F}$.

10.4. End of the proof. Now we can combine the previous bounds. Let $\eta>0$ and $\delta$ with $0<\delta<1 / 4$ be parameters to be determined later.

- If $M \leqslant q^{2 \delta}$, we then apply the bound (10.4) (and the dyadic decomposition of $T_{d, x}$ in a combination of sums $S_{d, M}$ ) to derive

$$
\sum_{d \leqslant D} T_{d, X} \ll X^{1+\varepsilon} q^{7 / 32-1}+Z^{10 / 9} X^{5 / 6+\varepsilon} q^{2 / 9+\delta / 3},
$$

under the condition that

$$
X \geqslant Z^{2 / 3} D^{2} q^{4 / 3+2 \delta}
$$

(see (10.3)).

- If $M \geqslant q^{1 / 2+\eta}$, we apply the bound (10.5) and sum over $d \leqslant D$ to find that

$$
\sum_{d \leqslant D} T_{d, X} \ll X^{1+\varepsilon}\left(\frac{1}{q^{1 / 2}}+\frac{q^{1 / 2}}{M}\right) \ll X^{1+\varepsilon} q^{-\eta}
$$

in that case. 
- If $q^{2 \delta} \leqslant M<q^{1 / 2+\eta}$, we then apply the bound (10.9) and sum over $d \leqslant D$, obtaining

$$
\sum_{d \leqslant D} T_{d, X} \ll X^{1+\varepsilon}\left(\frac{1}{q^{\delta}}+\frac{1}{q^{1 / 4}}+\frac{q^{1 / 2+\eta / 2}}{X^{1 / 2}}\right) \ll X^{1+\varepsilon}\left(q^{-\delta}+\frac{q^{1 / 2+\eta / 2}}{X^{1 / 2}}\right) .
$$

This covers all of the ranges for $M$. We now choose $\eta, \delta>0$ such that the bound in (10.12) is equal to the second term in (10.13) and the first term in (10.13) is consistent with the second term in (10.10). That is, we choose $q^{\eta}=(X / q)^{1 / 3}$ and $q^{\delta}=\frac{X^{1 / 8}}{Z^{5 / 6} q^{1 / 6}}$. Therefore, we have in all cases, the estimate

$$
\sum_{d \leqslant D} T_{d, X} \ll X^{2 / 3+\varepsilon} q^{1 / 3}+Z^{5 / 6} X^{7 / 8+\varepsilon} q^{1 / 6}+X^{1+\varepsilon} q^{7 / 32-1},
$$

for any $\varepsilon>0$, under the assumption that

$$
X \gg D^{8 / 3} q^{4 / 3} Z^{-4 / 3},
$$

and the implied constant depends only on $\varepsilon$ and the conductor of $\mathcal{F}$.

Finally, we combine this with the previously noted estimate

$$
\sum_{d>D} T_{d, X} \ll \frac{\|K\|_{\infty} X^{1+\varepsilon}}{D}
$$

(recall that for a nonexceptional trace function, we have $\|\widehat{K}\|_{\infty} \ll 1$ where the implied constant depends only on the conductor of $\mathcal{F}$ ) to conclude that

$$
\sum_{n \geqslant 1} \lambda(n)^{2} K(n) V\left(\frac{n}{X}\right) \ll X^{2 / 3+\varepsilon} q^{1 / 3}+Z^{5 / 6} X^{7 / 8+\varepsilon} q^{1 / 6}+X^{1+\varepsilon} / D .
$$

We take $D=q^{\gamma}$ for some small $\gamma>0$, and then we have

$$
\sum_{n \geqslant 1} \lambda(n)^{2} K(n) V\left(\frac{n}{X}\right) \ll X^{2 / 3+\varepsilon} q^{1 / 3}+Z^{5 / 6} X^{7 / 8+\varepsilon} q^{1 / 6}+X^{1+\varepsilon} q^{-\gamma},
$$

provided that

$$
X \gg q^{4 / 3+8 \gamma / 3} / Z^{4 / 3},
$$

where the implied constant depends only on $\varepsilon$ and the conductor of $\mathcal{F}$.

This concludes the proof of Corollary 1.6. 


\section{Acknowledgements}

YL, PM and EK were partially supported by a DFG-SNF lead agency program grant (grant number 200020L_175755). WS was partially supported by the Clay Mathematics Institute. We are very grateful to R. Holowinsky and P. Nelson for sharing with us and explaining their work [12] which has directly inspired the present paper. We are also very grateful to the referees for their careful reading of the manuscript, comments and suggestions and in particular for pointing out a serious error in the first version of Corollary 1.6.

\section{Conflict of Interest: None.}

\section{References}

[1] K. Aggarwal, R. Holowinsky, Y. Lin and Q. Sun, 'The Burgess bound via a trivial delta method', Ramanujan J. (2018), (to appear) arXiv:1803.00542.

[2] V. Blomer, 'Subconvexity for twisted L-functions on GL(3)', Amer. J. Math. 134(5) (2012), 1385-1421.

[3] J. B. Conrey and H. Iwaniec, 'The cubic moment of central values of automorphic $L$ functions', Ann. of Math. (2) 151(3) (2000), 1175-1216.

[4] É. Fouvry, E. Kowalski and Ph. Michel, 'Algebraic trace functions over the primes', Duke Math. J. 163(9) (2014), 1683-1736.

[5] É. Fouvry, E. Kowalski and Ph. Michel, Trace Functions Over Finite Fields and Their Applications, Colloquium De Giorgi 2013 and 2014, Colloquia, 5 (Ed. Norm., Pisa, 2014), 7-35.

[6] É. Fouvry, E. Kowalski and Ph. Michel, 'Algebraic twists of modular forms and Hecke orbits', GAFA 25(2) (2015), 580-657.

[7] É. Fouvry, E. Kowalski and Ph. Michel, 'On the exponent of distribution of the ternary divisor function', Mathematika 61(1) (2015), 121-144.

[8] É. Fouvry, E. Kowalski, Ph. Michel, C. Raju, J. Rivat and K. Soundararajan, 'On short sums of trace functions', Ann. Inst. Fourier 67 (2017), 423-449.

[9] J. B. Friedlander and H. Iwaniec, 'Incomplete Kloosterman sums and a divisor problem', Ann. of Math. (2) 121(2) (1985), 319-350. (with an appendix by B. J. Birch and E. Bombieri).

[10] D. Goldfeld, Automorphic Forms and L-Functions for the Group GL(, $\mathbf{R})$, Cambridge Studies in Advanced Mathematics, 99 (Cambridge University Press, Cambridge, 2006), With an appendix by Kevin A. Broughan.

[11] R. Holowinsky, R. Munshi and Z. Qi, 'Character sums of composite moduli and hybrid subconvexity', in Advances in the Theory of Automorphic Forms and Their L-functions, Contemporary Mathematics, 664 (American Mathematical Society, Providence, RI, 2016), 135-148.

[12] R. Holowinsky and P. Nelson, 'Subconvex bounds on GL(3) via degeneration to frequency zero', Math. Ann. 372(1-2) (2018), 299-319.

[13] H. Iwaniec, Topics in Classical Automorphic Forms, Graduate Studies in Mathematics, 17 (American Mathematical Society, Providence, RI, 1997). 
[14] N. M. Katz, Exponential Sums and Differential Equations, Annals of Mathematics Studies, 124 (Princeton University Press, Princeton, NJ, 1990).

[15] H. Kim and P. Sarnak, 'Refined estimates towards the Ramanujan and Selberg conjectures', J. Amer. Math. Soc. 16(1) (2003), 139-183. Appendix to H. Kim, Functoriality for the exterior square of $\mathrm{GL}_{4}$ and the symmetric fourth of $\mathrm{GL}_{2}$.

[16] E. Kowalski, Ph. Michel and W. Sawin, Stratification and averaging for exponential sums: Bilinear forms with generalized Kloosterman sums, Ann. Sc. Norm. Super. Pisa, (to appear), Preprint, 2018, arXiv:1802.09849.

[17] Y. Lin, 'Bounds for twists of GL(3) L-functions', Preprint, 2018, arXiv:1802.05111.

[18] S. D. Miller, 'Cancellation in additively twisted sums on GL(n)', Amer. J. Math. 128(3) (2006), 699-729.

[19] G. Molteni, 'Upper and lower bounds at $s=1$ for certain Dirichlet series with Euler product', Duke Math. J. 111(1) (2002), 133-158.

[20] R. Munshi, 'The circle method and bounds for $L$-functions-IV: Subconvexity for twists of GL(3) L-functions', Ann. of Math. (2) 182(2) (2015), 617-672.

[21] R. Munshi, 'Twists of GL(3) L-functions', Preprint, 2016, arXiv:1604.08000.

[22] I. Petrow and M. Young, 'The Weyl bound for Dirichlet $L$-functions of cube-free conductor', Preprint, 2018, arXiv:1811.02452.

[23] Q. Sun and R. Zhao, 'Bounds for $\mathrm{GL}_{3}$ L-functions in depth aspect', Forum Math. 31(2) (2019), 303-318.

[24] R. Zacharias, 'Simultaneous non-vanishing for Dirichlet L-functions', Ann. Inst. Fourier 69(4) (2019), 1459-1524.

[25] F. Zhou, 'The Voronoi formula on $G L(3)$ with ramification', Preprint, 2018, arXiv:1806.10786. 